\title{
A STABLE PENALTY METHOD FOR THE COMPRESSIBLE NAVIER-STOKES EQUATIONS: II. ONE-DIMENSIONAL DOMAIN DECOMPOSITION SCHEMES*
}

\author{
J. S. HESTHAVEN ${ }^{\dagger}$
}

\begin{abstract}
This paper presents asymptotically stable schemes for patching of nonoverlapping subdomains when approximating the compressible Navier-Stokes equations given on conservation form. The scheme is a natural extension of a previously proposed scheme for enforcing open boundary conditions and as a result the patching of subdomains is local in space. The scheme is studied in detail for Burgers's equation and developed for the compressible Navier-Stokes equations in general curvilinear coordinates.

The versatility of the proposed scheme for the compressible Navier-Stokes equations is illustrated for quasi-one-dimensional transonic nozzle flows and for flows around an infinitely long circular cylinder.
\end{abstract}

Key words. domain decomposition, stable penalty methods, Navier-Stokes equations

AMS subject classifications. 65M55, 65M70, 65M12, 35Q30

PII. S1064827594276540

1. Introduction. In the last decade, spectral collocation methods have been applied extensively for solving nonshocked nonlinear partial differential equations, often yielding results superior to those obtained by other methods. However, as the position of the grid points in the computational grid is predetermined, it has proven difficult to apply spectral methods to problems in complex geometries. For this reason, domain decomposition techniques, where the full computational domain is constructed by several geometrically simple subdomains, have been given increased attention. Besides the geometric flexibility, the multidomain approach also allows for efficiently resolving strongly localized features without overresolving smooth regions of the solution, and the approach lends itself to parallelization on contemporary parallel computer architectures with distributed memory.

In part I of this paper [1], we developed a novel set of open boundary conditions for the compressible Navier-Stokes equations leading to a well-posed continuous problem. We then proposed a penalty method to implement these boundary conditions and were able to prove asymptotic stability of the semidiscrete scheme when using a Legendre collocation method as the spatial approximation scheme. Through several numerical experiments we showed that these results carry over to Chebyshev collocation methods. The purpose of part II is to extend the use of these boundary conditions to act as local patching conditions when doing multidomain simulations of the unsteady, compressible Navier-Stokes equations. The emphasis will be on essentially one-dimensional patching schemes. However, by writing the Navier-Stokes equations in general curvilinear coordinates we show that the scheme is applicable also to multidimensional problems when patching is required along one general coordinate axis only. As a way of explaining the scheme, we first develop it for Burgers's equa-

${ }^{*}$ Received by the editors November 4, 1994; accepted for publication (in revised form) September 12, 1995. This work was partially supported by the Danish Computing Center for Research and Education $(\mathrm{UNI} \bullet \mathrm{C})$ and by the Danish Science Academy.

http://www.siam.org/journals/sisc/18-3/27654.html

${ }^{\dagger}$ Ris $\varnothing$ National Laboratory, Optics and Fluid Dynamics Department, P.O. Box 49, DK-4000 Roskilde, Denmark. Current address: Division of Applied Mathematics, Brown University, Box F, Providence, RI 02912 (jansh@cfm.brown.edu). 
tion and exemplify the method for Navier-Stokes equations by quasi-one-dimensional transonic Laval flows and two-dimensional unsteady flows around a circular cylinder.

The development of spectral multidomain methods has traditionally been centered around schemes for incompressible flows. Examples of such methods are the spectral element method [2] and the flux conservation method [3]. For a general review of alternative methods for incompressible flows we refer to [4]. For compressible flows, the emphasis until recently has been on methods for the inviscid Euler equations, where noniterative schemes [5,6], iterative schemes [7], and spectral element methods $[8,9]$ have been proposed. For viscous compressible flows, several methods have recently appeared $[10,11,12]$ although the emphasis has been on methods for steady state problems. All previous methods for viscous flows are based on a treatment of the inviscid part of the equation, in most cases by applying methods known from the Euler equations, and a separate treatment of the viscous part of the equation. This second contribution is then applied as a correction to the result obtained from the inviscid patching.

The main difference between previously proposed methods and the one introduced here is that we develop a patching scheme which accounts for the inviscid and viscous part of the equation simultaneously. This approach is made possible by implementing the interface conditions using a penalty term, hence allowing for boundary conditions of a general type. In the inviscid limit the proposed algorithm is shown to be equivalent to schemes known from the Euler equations. Emphasis is directed toward developing methods that can handle general unsteady flows and we apply high-order explicit time integration schemes to verify that the proposed methods are well suited for simulating unsteady flows.

The paper is organized as follows. In section 2 we review some well-known results on Chebyshev polynomials and collocation methods which will be used extensively in the remaining part of the paper. We have also included a more detailed discussion on the advantages of domain decomposition. This leads to section 3 where we develop a multidomain scheme for solving Burgers's equation, study convergence, and compare the performance of the proposed scheme with that of alternative multidomain methods. Section 4 develops a novel scheme for performing one-dimensional patching of nonoverlapping domains when solving the compressible Navier-Stokes equations in general curvilinear coordinates. The scheme is based on a penalty method approach, and we argue that the global scheme is asymptotically stable. As examples of the performance of the scheme, we compute quasi-one-dimensional transonic nozzle flows at medium Reynolds numbers $(\mathrm{Re} \leq 1000)$ and unsteady flow around an infinitely long circular cylinder. Section 5 contains a brief conclusion and suggestions for future work.

2. General concepts. Prior to discussing the multidomain schemes we will review a few well-known results, which will be useful in the following sections.

2.1. Chebyshev polynomials and collocation methods. The schemes presented in this paper are all based on Chebyshev collocation methods, which, due to their superior approximation properties, are widely used when solving nonlinear partial differential equations. This choice is not unique and the methods proposed in this paper may be used equally well in connection with other classical polynomial families or even finite difference schemes.

The Chebyshev polynomial of order $k$ is defined as

$$
T_{k}(x)=\cos \left(k \cos ^{-1} x\right),
$$


where $|x| \leq 1$. In the following sections we will consider collocation methods, where the $N+1$ collocation points are chosen to be the Chebyshev-Gauss-Lobatto points found as the roots of the polynomial $\left(1-x^{2}\right) T_{N}^{\prime}(x)$, i.e.,

$$
x_{i}=\div \cos \left(\frac{i \pi}{N}\right), \quad 0 \leq i \leq N .
$$

Associated with the Gauss-Lobatto points is a quadrature formula, stating that for a polynomial $f(x)$ of maximum degree $2 N-1$, one has the exact result

$$
\frac{\pi}{N} \sum_{i=0}^{N} \frac{f\left(x_{i}\right)}{c_{i}}=\int_{-1}^{1} \frac{f(\xi)}{\sqrt{1-\xi^{2}}} d \xi,
$$

where $c_{0}=c_{N}=2$ and $c_{i}=1$ for $1 \leq i \leq N-1$. The quadrature rule leads to the definition of the discrete weighted $L_{2}$-norm as we will use it in this paper:

$$
L_{2}(f)=\|f\|^{2}=\int_{-1}^{1} \frac{f^{2}(\xi)}{\sqrt{1-\xi^{2}}} d \xi \leq \frac{\pi}{N} \sum_{i=0}^{N} \frac{f^{2}\left(x_{i}\right)}{c_{i}} \leq 2\|f\|^{2} .
$$

Proof for the inequalities may be found in [13]. For further details on the properties of the Chebyshev polynomials, we refer to [14].

When applying a Chebyshev collocation method, the function $f(x)$ is approximated by a grid function $f_{i}=f\left(x_{i}\right)$, where the grid points are the Gauss-Lobatto points. We construct a global $N$ th-order Chebyshev interpolant $I_{N}$ to obtain the approximation of the function

$$
\left(I_{N} f\right)(x)=\sum_{i=0}^{N} f_{i} g_{i}(x)
$$

where the interpolating Chebyshev-Lagrange polynomials are given as

$$
g_{i}(x)=\frac{\left(1-x^{2}\right) T_{N}^{\prime}(x)(-1)^{i+1+N}}{c_{i} N^{2}\left(x-x_{i}\right)} .
$$

It is easily verified that by construction

$$
\left(I_{N} f\right)\left(x_{i}\right)=f_{i} .
$$

To seek approximate solutions $\left(I_{N} f\right)(x)$ to a partial differential equation, we need to obtain values of the spatial derivatives at the collocation points. This is accomplished by approximating the continuous differential operator by a matrix operator with the entries given as

$$
\mathcal{D}_{i j}=g_{j}^{\prime}\left(x_{i}\right),
$$

implying that the derivative of $f$ at a collocation point $x_{i}$ is approximated as

$$
\frac{d f}{d x}\left(x_{i}\right) \approx \frac{d\left(I_{N} f\right)}{d x}\left(x_{i}\right)=\sum_{j=0}^{N} \mathcal{D}_{i j} f\left(x_{j}\right)
$$

and likewise for higher derivatives. For the explicit expressions of the entries of the matrix operator and further details on collocation methods, we refer to [13, 14]. 
Filtering of the solution may be used for reasons of increased stability. In the implementation of the schemes to be presented later, we employ an exponential filter of the type

$$
\sigma_{i}=\left\{\begin{array}{cl}
1, & 0 \leq i \leq N_{c} \\
\exp \left[-\alpha\left(\frac{i-N_{c}}{N-N_{c}}\right)^{\gamma}\right], & N_{c}<i \leq N
\end{array}\right.
$$

where $N_{c}$ is a cutoff mode number, $\gamma$ is the order of the filter, and $\alpha=-\ln \varepsilon_{M}$ with $\varepsilon_{M}$ being the machine accuracy. This choice of filter function is by no means unique and alternatives may be found in [4]. The filtering may conveniently be expressed as a matrix operator $\mathcal{F}$ with the entries

$$
\mathcal{F}_{i j}=\frac{2}{c_{j} N} \sum_{k=0}^{N} \frac{\sigma_{k}}{c_{k}} T_{k}\left(x_{i}\right) T_{k}\left(x_{j}\right)
$$

We emphasize that the use of filters in the present work is motivated only by the request for a large time-step. The schemes to be presented all remain stable in the absence of filters, albeit at the expense of a smaller maximum time-step.

2.2. One domain versus multidomain collocation schemes. The high accuracy, which may be obtained when approximating smooth solutions using a onedomain spectral method, is gained at the cost of introducing several disadvantages. Since the collocation points are given a priori there is little room for local grid refinement through smooth grid mappings; see, e.g., [4]. Thus, if the solution contains strongly localized features we need a large number of modes $(N)$ in order to properly resolve such features. Since the evaluation of derivatives is an $\mathcal{O}\left(N^{2}\right)$ operation this results in significant computational requirements. In the case where an FFT may be used for this computation, the operation count reduces to $\mathcal{O}(N \log N)$. However, for the sake of simplicity we assume in the following that all derivatives are calculated using matrix-vector products as no qualitative differences are introduced in the discussion when using an FFT. For hyperbolic problems the Courant-Friedrichs-Lewy (CFL) condition requires $\Delta t \propto N^{-2}$ [15], resulting in a severe time-step restriction for explicit time-integration schemes at high resolution. Consequently, such problems are computationally intensive. Additionally, the high accuracy that one expects from such a scheme may not be obtained after all. As was recently shown [16, 17], the Chebyshev collocation differentiation matrix is very ill conditioned, leading to severe degradation of the results at high resolution due to roundoff errors. This is particularly true for problems with high-order spatial derivatives.

Most of these disadvantages may be diminished or even eliminated by applying a multidomain approach. In the present paper we split the computational domain $\Omega$ into $K$ nonoverlapping connected domains $\Omega^{k}$ such that $\Omega=\bigcup_{k=1}^{K} \Omega^{k}$ and the subdomain boundaries $\Gamma^{k}$ are defined as $\Gamma^{k}=\Omega^{k} \cap \Omega^{k+1}$ for $1 \leq k \leq K-1$. The outer boundaries are termed $\Gamma^{0}$ and $\Gamma^{K}$, respectively.

The advantages of this approach may be summarized as follows:

- Independent choice of resolution in each subdomain allows for treating strongly localized solutions without overresolving smooth regions of the solution.

- Splitting the domain into $K$ similar subdomains, each with $N / K$ modes, allows for using a time-step $\Delta t \propto K^{-1}(N / K)^{-2}$ as opposed to $\Delta t \propto N^{-2}$ when applying explicit time integration of hyperbolic problems. 
- The operations needed for evaluation of a global spatial derivative may be reduced to $\mathcal{O}\left(K(N / K)^{2}\right)$ as compared to $\mathcal{O}\left(N^{2}\right)$.

- The effect of round-off error when evaluating a first derivative may be reduced significantly from $\mathcal{O}\left(\varepsilon_{M} N^{2}\right)$ to $\mathcal{O}\left(\varepsilon_{M}(N / K)^{2}\right)$, with $\varepsilon_{M}$ being the machine accuracy $[16,17]$.

- Assuming that the patching of subdomains is local in space, the scheme is well suited for implementation on contemporary parallel computer architectures with distributed memory.

Through a variational argument, due to Gottlieb [18], we may gain further insight into the relation between accuracy and computational workload when considering a multidomain approach.

If we assume the solution to be smooth, we may expect the approximation error $E(N, K)$ to scale as

$$
E(N, K) \propto\left(\frac{\pi k}{K N}\right)^{N}
$$

where $k$ is the maximum wavenumber in the function and $N$ is the number of modes in each of the $K$ subdomains. When approximating the solution we require the maximum absolute error to be bounded as

$$
E=\exp (-\gamma) \leq \varepsilon
$$

The computational work required to compute, e.g., the flux of a variable in a timedependent problem, may be estimated as

$$
W(N, K)=c_{1} K N^{2}+c_{2} K N
$$

where $c_{1}$ and $c_{2}$ are problem-specific constants. Our aim is to minimize the work required to obtain a solution with a specified maximum error $\varepsilon$. Thus, by introducing a Lagrange multiplier $\lambda$ we construct the functional $F(N, K, \lambda)$ as

$$
F(N, K, \lambda)=c_{1} K N^{2}+c_{2} K N+\lambda\left[N \ln \left(\frac{\pi k}{K N}\right)+\gamma\right]
$$

From this we obtain (see, e.g., [19, Chap. IV]) the optimal values of $N$ and $K$ as

$$
N_{\mathrm{opt}}=\frac{1}{2}\left(\gamma+\sqrt{\gamma^{2}+4 \frac{c_{2}}{c_{1}}}\right) \simeq \gamma
$$

and

$$
K_{\mathrm{opt}}=\frac{\pi k}{N_{\mathrm{opt}}} \exp \left(\frac{\gamma}{N_{\mathrm{opt}}}\right) .
$$

One immediately observes that high accuracy may be obtained only by using a large number of modes $N$ and not, as one could expect, by applying many subdomains, each with a low number of modes. This reflects the well-known property that a minimum of $\pi$ modes is needed in a Chebyshev expansion to properly resolve a single wave [20]. We may conclude that for very smooth and regular solutions, where $k$ is small, a multidomain solution is not the method of choice. Contrary to that, if the solution exhibits strongly localized phenomena, i.e., $k$ is large, one should introduce several 
domains in order to minimize the computational work. When taking into account the effects of round-off error, decreasing the number of modes in each subdomain becomes even more important. Although these arguments are not very strict in nature they still lead to the observation that an optimal choice of $N$ and $K$ seems to be a few larger subdomains, each with a not too small number of modes in order to maintain high spatial accuracy. Similar observations were made by Wasberg [16] when comparing one-domain and multidomain Chebyshev approximation of simple functions. In the next section we shall return to these speculations and compare with actual numerical results obtained for Burgers's equation.

3. Burgers's equation. Let us consider Burgers's equation

$$
\frac{\partial U}{\partial t}+U \frac{\partial U}{\partial x}=\varepsilon \frac{\partial^{2} U}{\partial x^{2}},|x| \leq 1, \quad t>0,
$$

with $\varepsilon \geq 0$ and the initial condition

$$
U(x, 0)=f(x),
$$

with boundary conditions of the form

$$
\begin{aligned}
& \alpha U(-1, t)-\left.\beta \varepsilon \frac{\partial U}{\partial x}\right|_{x=-1}=g_{1}(t), \\
& \gamma U(1, t)+\left.\delta \varepsilon \frac{\partial U}{\partial x}\right|_{x=1}=g_{2}(t),
\end{aligned}
$$

where $\alpha, \beta, \gamma$, and $\delta$ are nonnegative constants.

As was shown in [21] and used extensively in [1], it is sufficient to consider the linearized, constant coefficient version of Burgers's equation

$$
\frac{\partial U}{\partial t}+U_{0} \frac{\partial U}{\partial x}=\varepsilon \frac{\partial^{2} U}{\partial x^{2}}, \quad|x| \leq 1, \quad t>0
$$

when addressing the issue of wellposedness of the problem. Here $U_{0}$ is the uniform solution around which we have linearized. result.

The four constants in (4) may not be chosen arbitrarily. We will use the following

LEMma 3.1. Assume there exists a solution $U$ to (5) subject to boundary conditions as given in (4). The problem is well posed if $\alpha, \beta, \gamma$, and $\delta$ are chosen as

$$
\begin{aligned}
& U_{0} \geq 0: \alpha=U_{0}, \quad \beta=1, \quad \gamma=0, \quad \delta=1, \\
& U_{0}<0: \alpha=0, \quad \beta=1, \quad \gamma=\left|U_{0}\right|, \quad \delta=1 .
\end{aligned}
$$

Proof. The proof follows directly from Lemma 3.1 in [1].

In [1] we found this choice of open boundary conditions to perform well and to maintain spectral accuracy. When considering a multidomain approach, the important observation to make is that we may equally well treat a subdomain boundary as an open boundary. However, it is a very special open boundary since we may obtain accurate boundary conditions from the neighboring subdomains. This observation leads to the scheme proposed in the following section. 
3.1. The multidomain scheme. We wish to solve (3) using a multidomain Chebyshev collocation method where the collocation points are the Chebyshev-GaussLobatto points. In each subdomain $\Omega^{k}$ this approach involves finding a $N^{k}$ th degree polynomial $u^{k}\left(x^{k}, t\right)$ satisfying

$$
\frac{\partial u^{k}}{\partial t}+u^{k} \frac{\partial u^{k}}{\partial x^{k}}=\varepsilon \frac{\partial^{2} u^{k}}{\partial\left(x^{k}\right)^{2}} \quad \text { at } x^{k}=x_{i}^{k}, \quad i \in\left[1, \ldots, N^{k}-1\right],
$$

in the interior, where $x_{i}^{k} \in \Omega^{k}$ are the collocation points in the $k$ th subdomain with $N^{k}$ modes. The boundary operator in each subdomain is given as

$$
\begin{aligned}
& \alpha u_{0}^{k}-\beta \varepsilon \frac{\partial u_{0}^{k}}{\partial x^{k}}=g_{1}^{k}(t), \\
& \gamma u_{N}^{k}+\delta \varepsilon \frac{\partial u_{N}^{k}}{\partial x^{k}}=g_{2}^{k}(t),
\end{aligned}
$$

where $g_{1}^{k}(t)$ and $g_{2}^{k}(t)$ are the boundary conditions at each subdomain boundary and we have introduced the symbol $u_{i}^{k}=u^{k}\left(x_{i}^{k}, t\right)$.

The penalty method, as introduced in [1], for the implementation of open boundary conditions generalizes straightforwardly to the multidomain approach, leading us to propose the following scheme for a multidomain solution of Burgers's equation:

$$
\begin{aligned}
\forall k: \frac{\partial u^{k}}{\partial t}+u^{k} \frac{\partial u^{k}}{\partial x^{k}}= & \varepsilon \frac{\partial^{2} u^{k}}{\partial\left(x^{k}\right)^{2}} \\
& -\tau_{1}^{k} Q_{k}^{-}\left(x_{i}^{k}\right)\left[\alpha u_{0}^{k}-\beta \varepsilon \frac{\partial u_{0}^{k}}{\partial x^{k}}-g_{1}^{k}(t)\right] \\
& -\tau_{2}^{k} Q_{k}^{+}\left(x_{i}^{k}\right)\left[\gamma u_{N}^{k}+\delta \varepsilon \frac{\partial u_{N}^{k}}{\partial x^{k}}-g_{2}^{k}(t)\right],
\end{aligned}
$$

where

$$
Q_{k}^{-}\left(x_{i}^{k}\right)=\delta_{i 0}, \quad Q_{k}^{+}\left(x_{i}^{k}\right)=\delta_{i N^{k}} .
$$

Here $\delta_{i j}$ is the Kronecker delta function with subscript $i$ corresponding to the collocation point $x_{i}^{k}$. Thus, the two penalty terms are zero except at the two boundary points. We now need to specify the boundary conditions $g_{1}^{k}(t)$ and $g_{2}^{k}(t)$ at the subdomain boundaries. Following the result in Lemma 3.1, we observe that the type of boundary condition depends on the sign of $U_{0}$. The general form of the boundary conditions at the subdomain boundaries becomes

$$
\begin{aligned}
& g_{1}^{k}(t)=\alpha u_{N}^{k-1}-\beta \varepsilon \frac{\partial u_{N}^{k-1}}{\partial x^{k-1}}, \\
& g_{2}^{k}(t)=\gamma u_{0}^{k+1}+\delta \varepsilon \frac{\partial u_{0}^{k+1}}{\partial x^{k+1}} .
\end{aligned}
$$

At the two limiting boundaries $\Gamma^{0}$ and $\Gamma^{K}$, the boundary conditions are obtained from (4), i.e.,

$$
g_{1}^{1}(t)=g_{1}(t), \quad g_{2}^{K}(t)=g_{2}(t) .
$$

Hence, the patching scheme begins by determining the sign of $U_{0}^{k}$. In the examples to be presented later, we have used $u^{k}$ at the boundary, obtained at the previous timestep, to linearize around, i.e., $U_{0}^{k}=u^{k}\left(\Gamma^{k}, t\right)$. Once the sign of $U_{0}^{k}$ is determined, 
the proper type of boundary operator is found through Lemma 3.1, and information is taken from the neighboring subdomain to calculate the appropriate value at the boundary point.

For simplicity, consider the boundary $\Gamma^{k}$ and assume that $U_{0}^{k}>0$. The equation solved at $\Gamma^{k}$ in the left domain $\Omega^{k}$ becomes

$$
\frac{\partial u_{N}^{k}}{\partial t}+u_{N}^{k} \frac{\partial u_{N}^{k}}{\partial x^{k}}=\varepsilon \frac{\partial^{2} u_{N}^{k}}{\partial\left(x^{k}\right)^{2}}-\tau_{2}^{k} \varepsilon\left(\frac{\partial u_{N}^{k}}{\partial x^{k}}-\frac{\partial u_{0}^{k+1}}{\partial x^{k+1}}\right) .
$$

Likewise, the equation solved at $\Gamma^{k}$ in the right domain $\Omega^{k+1}$ yields

$$
\begin{aligned}
\frac{\partial u_{0}^{k+1}}{\partial t}+u_{0}^{k+1} \frac{\partial u_{0}^{k+1}}{\partial x^{k+1}}= & \varepsilon \frac{\partial^{2} u_{0}^{k+1}}{\partial\left(x^{k+1}\right)^{2}} \\
& -\tau_{1}^{k+1}\left(U_{0}^{k}\left(u_{0}^{k+1}-u_{N}^{k}\right)-\varepsilon\left(\frac{\partial u_{0}^{k+1}}{\partial x^{k+1}}-\frac{\partial u_{N}^{k}}{\partial x^{k}}\right)\right) .
\end{aligned}
$$

Thus, the patching enforces continuity of the function $u$ and its first derivative across the subdomain boundary, but only in a weak sense. Nevertheless, as we shall see shortly, the global spectral accuracy of the multidomain scheme is maintained.

To complete the description of the algorithm, we need to determine $\tau_{1}^{k}$ and $\tau_{2}^{k}$ such that the overall scheme is stable. However, we observe that the scheme in each subdomain is equivalent to the one-domain scheme proposed in [1]. Thus, asymptotic stability of the scheme in each subdomain may be assured by choosing $\tau_{1}^{k}$ and $\tau_{2}^{k}$ as derived in [1], where details of the one-domain proof may be found. Hence, asymptotic stability is ensured provided

$$
\begin{aligned}
\forall k: \tau_{1}^{k} & =\frac{1}{\omega \varepsilon \beta}\left[\varepsilon+2 \kappa_{1}-2 \sqrt{\kappa_{1}^{2}+\varepsilon \kappa_{1}-1 / 2 \varepsilon \omega\left|U_{0}^{k}\right|}\right] \frac{2}{L_{k}}, \\
\tau_{2}^{k} & =\frac{1}{\omega \varepsilon \delta}\left[\varepsilon+2 \kappa_{2}-2 \sqrt{\kappa_{2}^{2}+\varepsilon \kappa_{2}-1 / 2 \varepsilon \omega\left|U_{0}^{k+1}\right|}\right] \frac{2}{L_{k}},
\end{aligned}
$$

where

$$
\omega=\left(\frac{1}{N^{k}}\right)^{2}, \quad \kappa_{1}=\frac{\alpha \omega}{\beta}, \quad \kappa_{2}=\frac{\gamma \omega}{\delta},
$$

and $L_{k}$ is the length of the $k$ th subdomain. Reduced expressions for the penalty parameters for different choices of $\alpha, \beta, \gamma$, and $\delta$ are given in [1]. In the actual implementation of the scheme, it is possible to reduce the parameters given in (8) by a factor of four while maintaining stability, as discussed extensively in [1]. This allows for time-stepping the scheme with an optimal time-step and results in the parameters being used in all numerical tests presented in the following paragraphs.

In the following examples we have employed an adaptive fourth order RungeKutta method for time-stepping, with the global time-step $\Delta t$ being calculated as

$$
\Delta t=\mathrm{CFL} \times \min _{i, k}\left[\frac{\left|u_{i}^{k}\right|}{\Delta_{i} x^{k}}+\frac{\varepsilon}{\left(\Delta_{i} x^{k}\right)^{2}}\right]^{-1},
$$

where $\Delta_{i} x^{k}=x_{i}^{k}-x_{i-1}^{k}$ is the local grid spacing and $u_{i}^{k}=u^{k}\left(x_{i}^{k}, t\right)$. The boundary conditions and the subdomain patching are enforced at the intermediate time-steps in 
the Runge-Kutta method, and we enforce continuity of the global solution. Following each complete cycle of the Runge-Kutta scheme, we apply a high-order exponential filter as given from (2) in each subdomain in order to slightly smooth the global solution. We recall that the filter is applied only to increase the maximum allowable time-step as the scheme remains stable also in the absence of the filter. In all test cases presented later, the use of the filter allows for an average two-fold increase of the maximum time-step.

Although the scheme may seem rather complicated at first, a few things are useful to realize. As we are using spectral methods, the derivatives at the boundaries are calculated when evaluating the flux at the interior points. Thus, very few computations are required to perform the patching as described by (6)-(8) if the gradients at the boundary points $\Gamma^{k}$ are stored while calculating the flux. Additionally, since all calculations in the subdomains are independent and the patching is local in space and time, the scheme is well suited for parallelization. This may not be of importance for simple one-dimensional problems as the Burgers's equation. However, for more complex sets of equations, like the compressible Navier-Stokes equations, this is of significant importance, particularly when addressing multidimensional problems.

3.2. Numerical tests. In order to assess the accuracy of the proposed scheme, we have performed detailed studies of the evolution of two separate initial conditions to Burgers's equation. In the first part of this section we investigate the convergence properties of the scheme for the traveling wave solution and discuss further the problem of an optimal choice of number of modes and subdomains as mentioned in section 2.2. Following that, we compare the performance of the scheme with that of alternative multidomain methods for a viscous stationary shock.

3.2.1. Traveling wave solution. Burgers's equation, (3), has a rightward traveling wave solution (see, e.g., [21]) of the form

$$
U(x, t)=-a \tanh \left(a \frac{x-c t}{2 \varepsilon}\right)+c, x \in[-\infty, \infty], t \geq 0,
$$

where the free-stream values

$$
\lim _{x \rightarrow-\infty} U(x, t)=b_{-\infty}, \quad \lim _{x \rightarrow \infty} U(x, t)=b_{\infty}
$$

are associated with the wave speed $c$ and the constant $a \geq 0$ as

$$
c=\frac{b_{-\infty}+b_{\infty}}{2}, a=\frac{b_{-\infty}-b_{\infty}}{2} .
$$

Since we know the exact solution, the boundary conditions (4) at the outer boundaries may be given exactly at all times and we use the free-stream values $b_{-\infty}$ and $b_{\infty}$ to linearize around.

We have employed a standard Chebyshev collocation method in each subdomain when implementing the multidomain approach discussed in the previous section. In Fig. 1 we show a four-domain solution at equidistant times with $N=8, L=0.5$ in each subdomain, and $\varepsilon=0.1$. This clearly demonstrates the ability of the proposed scheme to perform the patching, and we observe no spurious reflections from the subdomain boundaries. Here, like in all subsequent simulations of Burgers's equation, we have applied an exponential filter (2) to the solution with $N_{c}=N / 2$ and $\gamma=6$. 


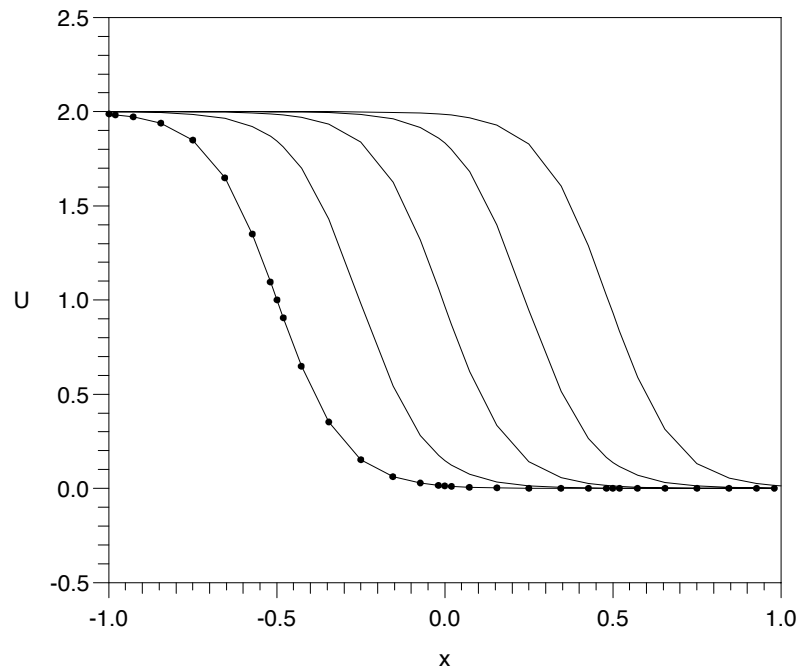

FIG. 1. Four-domain solution of the traveling wave solution to Burgers's equation with $b_{-\infty}=$ $2.0, b_{\infty}=0.0$, and $\varepsilon=0.1$. The dots at the initial wave represent the collocation points in each subdomain. The waves are separated by 0.25 in time.

(a)

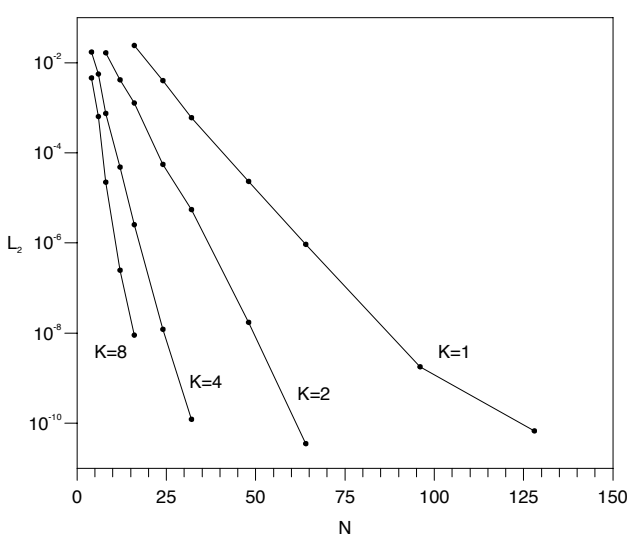

(b)

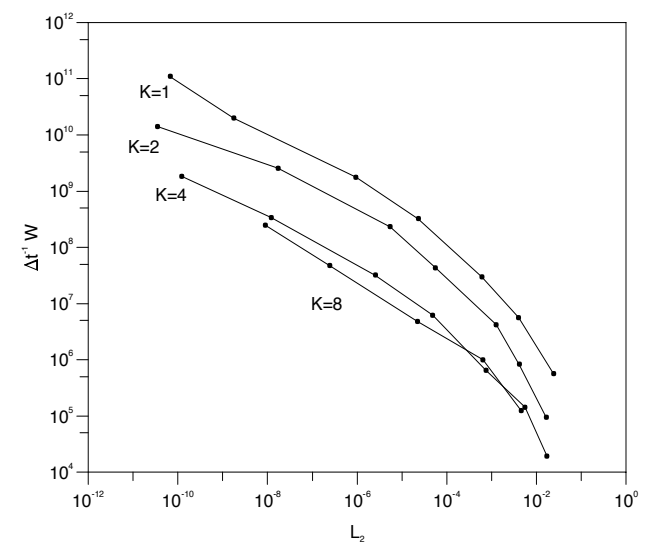

FIG. 2. (a) The global $L_{2}$-error (1) of the traveling wave solution to Burgers's equation with parameters as in Fig. 1. The solution is obtained for various combinations of numbers of subdomains $(K)$ and modes in each subdomain $(N)$. (b) Estimated number of operations needed to integrate Burgers's equation to $T=1.0$ for combinations of $K$ and $N$ as a function of the global $L_{2}$-error (1) at $T=1.0 . \Delta t$ is found using (9) with $C F L=3.0$.

In order to study the convergence properties of the proposed scheme, we have done a large number of simulations with varying number of subdomains $K$ and number of modes in each subdomain $N$.

We clearly observe in Fig. 2(a), where we plot the global $L_{2}$-error (1) as a function of $K$ and $N$, that the multidomain scheme preserves the spectral convergence. In the terminology of finite elements (see, e.g., [22]), we observe that the scheme preserves global spectral $p$-convergence (constant $K$ in Fig. 2(a)) as well as $h$-convergence (constant $N$ in Fig. 2(a)). 
In section 2.2 we briefly discussed how to choose the optimal number of subdomains and number of modes in each subdomain. We observed that if very high spatial accuracy is required only few domains with many modes should be used. This is clearly confirmed by the results in Fig. 2(a). However, we note that for the one domain solution $K=1$ the convergence rate decays for very large $N$. This is a consequence of round-off error caused by ill conditioning of the Chebyshev differentiation matrix [17].

When considering time-dependent problems, it is natural also to take into account the maximum allowable time-step while considering the optimal choice of $K$ and $N$. When using a fourth-order Runge-Kutta method with a filtered solution, we estimate the work for advancing one time-step to be

$$
W \sim 9 K N^{2}+21 K N
$$

In Fig. 2(b) we plot $\Delta t^{-1} W$ as an estimate of the work needed for advancing to $T=1.0$ as a function of the global $L_{2}$-error (1) at $T=1.0$. This figure clearly illustrates the existence of an optimal choice of $K_{\mathrm{opt}} \simeq 4-8$ unless very high accuracy is required. This conforms well with the results quoted in section 2.2 .

3.2.2. Stationary viscous shock. We now consider the dynamical evolution of Burgers's equation subject to Dirichlet boundary conditions

$$
U( \pm 1, t)=0,
$$

with the initial conditions being

$$
U(x, 0)=-\sin (\pi x)
$$

and $\varepsilon=0.01 / \pi$ in (3). For this initial condition, the wave steepens to a sawtooth wave with a very sharp, although smooth, profile centered around $x=0.0$. There exists an analytical solution to this problem [23], thus allowing for tests of the ability of the scheme to resolve sharp gradients.

This test case has previously been used as a benchmark for validating various discretization methods, including one-domain Fourier, Chebyshev, and finite difference methods [23], spectral element methods [23, 24], and an alternative, nonoverlapping local spectral multidomain method [12].

Here we choose to split the computational domain into four subdomains given as $\Omega^{1}=[-1.0,-0.05], \Omega^{2}=[-0.05,0.0], \Omega^{3}=[0.0,0.05]$, and $\Omega^{4}=[0.05,1.0]$ and with the same number of modes $N$ in each subdomain. This splitting of the domain is equivalent to what was used in $[12,23]$. In Fig. 3(a) we show the evolution of the initial condition at equidistant times. We note that the profile remains smooth due to the fine resolution around the sharp gradient. In Fig. 3(b) we show the calculated gradient of the solution around $x=0.0$ and observe that also the gradient of the solution remains smooth and continuous across the subdomain boundaries.

By monitoring the maximum gradient of the viscous shock we may judge the ability of the multidomain scheme to accurately capture this. In Table 1 we compare the calculated maximum value of $\partial u / \partial x$ and the time at which it occurs with that obtained from the analytical solution and from using one-domain Chebyshev methods.

We observe excellent agreement with the analytic solution, confirming that the scheme maintains spectral convergence of the gradient, despite the use of several domains. Comparing the accuracy by which we find the gradient and the correct time with that found by other methods [12, 23, 24], it is clear that the scheme proposed here 
(a)

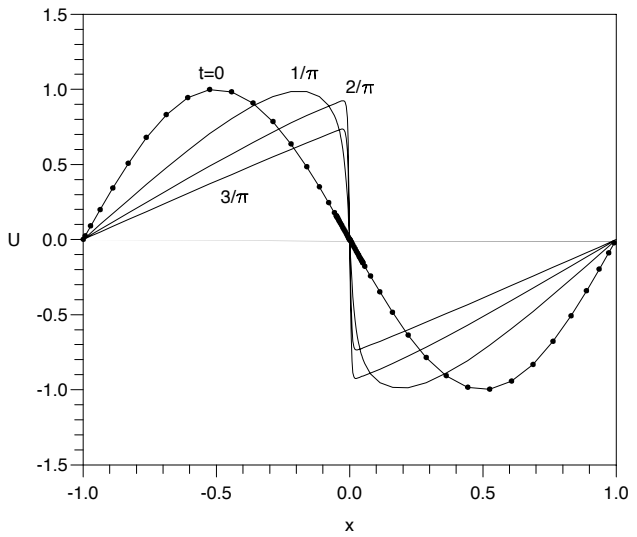

(b)

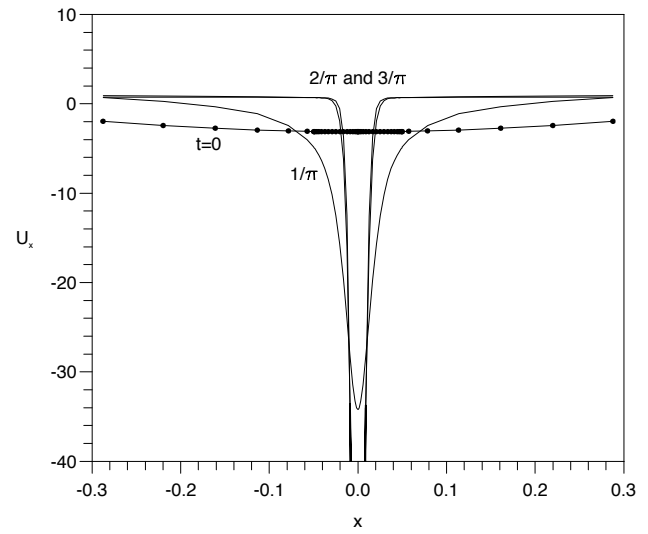

FIG. 3. (a) Viscous shock solution of Burgers's equation. The dots on the initial conditions represent the collocation points in the four-domain solution. (b) First derivative of the four-domain solution shown in (a).

TABLE 1

Comparison of the accuracy of the solution of the viscous shock problem. Here $N$ is the number of Chebyshev modes in each of the $K$ subdomains. The gradient of the shock is monitored, and the maximum steepness, $\left.\frac{\partial u}{\partial x}\right|_{\max }$, is found at $t_{\max }$. The schemes have been time-stepped with a fourth-order Runge-Kutta method with $C F L=3.0$ in (9). The marked numbers are obtained from [23].

\begin{tabular}{||l|c||r|r||}
\hline \multicolumn{1}{|c||}{$N$} & $K$ & $\left.\frac{\partial u}{\partial x}\right|_{\max }$ & $\pi t_{\max }$ \\
\hline 128 & 1 & -68.86944 & 1.5807 \\
256 & 1 & -112.76781 & 1.5952 \\
$512^{*}$ & 1 & -145.87700 & 1.6000 \\
\hline 10 & 4 & -155.67851 & 1.6339 \\
12 & 4 & -150.27248 & 1.6039 \\
14 & 4 & -151.63657 & 1.5983 \\
16 & 4 & -152.03909 & 1.6026 \\
18 & 4 & -152.00051 & 1.6041 \\
24 & 4 & -152.00438 & 1.6037 \\
\hline \multicolumn{2}{|l||}{ Analytic* } & -152.00516 & 1.6037 \\
\hline
\end{tabular}

performs at least as well as all other methods, and in some cases significantly better. It is also clear that resolving such a strong gradient using a one-domain method requires a very large number of modes, thus rendering such an approach ill suited for practical purposes.

4. The compressible Navier-Stokes equation. In this section we develop a multidomain scheme for the three-dimensional, compressible Navier-Stokes equations on conservation form. Although we consider the three-dimensional case, we require at present that the patching is done along one general coordinate axis only.

Consider the nondimensional, compressible Navier-Stokes equations in general curvilinear coordinates 


$$
\frac{\partial \overline{\mathbf{q}}}{\partial t}+\frac{\partial \overline{\mathbf{F}}}{\partial \xi}+\frac{\partial \overline{\mathbf{G}}}{\partial \eta}+\frac{\partial \overline{\mathbf{H}}}{\partial \zeta}=\frac{1}{\operatorname{Re}_{\mathrm{ref}}}\left(\frac{\partial \overline{\mathbf{F}}_{\nu}}{\partial \xi}+\frac{\partial \overline{\mathbf{G}}_{\nu}}{\partial \eta}+\frac{\partial \overline{\mathbf{H}}_{\nu}}{\partial \zeta}\right) .
$$

The curvilinear coordinates are defined as

$$
\xi=\xi(x, y, z), \quad \eta=\eta(x, y, z), \quad \zeta=\zeta(x, y, z),
$$

and are related to the Cartesian coordinates $(x, y, z)$ through the Jacobian transformation

$$
J=\left|\frac{\partial(x, y, z)}{\partial(\xi, \eta, \zeta)}\right|
$$

The state vector $\overline{\mathbf{q}}$ and the inviscid flux vectors are defined

$$
\overline{\mathbf{q}}=J \mathbf{q},
$$

and

$$
\begin{aligned}
& \overline{\mathbf{F}}=J\left(\mathbf{F} \xi_{x}+\mathbf{G} \xi_{y}+\mathbf{H} \xi_{z}\right), \\
& \overline{\mathbf{G}}=J\left(\mathbf{F} \eta_{x}+\mathbf{G} \eta_{y}+\mathbf{H} \eta_{z}\right), \\
& \overline{\mathbf{H}}=J\left(\mathbf{F} \zeta_{x}+\mathbf{G} \zeta_{y}+\mathbf{H} \zeta_{z}\right),
\end{aligned}
$$

where

$$
\mathbf{q}=\left[\begin{array}{c}
\rho \\
\rho u \\
\rho v \\
\rho w \\
E
\end{array}\right], \quad \mathbf{F}=\left[\begin{array}{c}
\rho u \\
\rho u^{2}+p \\
\rho u v \\
\rho u w \\
(E+p) u
\end{array}\right], \quad \mathbf{G}=\left[\begin{array}{c}
\rho v \\
\rho u v \\
\rho v^{2}+p \\
\rho v w \\
(E+p) v
\end{array}\right], \quad \mathbf{H}=\left[\begin{array}{c}
\rho w \\
\rho u w \\
\rho v w \\
\rho w^{2}+p \\
(E+p) w
\end{array}\right]
$$

Here $\rho$ is the density, $u, v, w$ are the three Cartesian velocity components, $E$ is the total energy, and $p$ is the pressure. In the following we will use $(x, y, z)$ and $\left(x_{1}, x_{2}, x_{3}\right)$ interchangeably to denote the Cartesian coordinates and likewise we use both $(u, v, w)$ and $\left(u_{1}, u_{2}, u_{3}\right)$ to identify the Cartesian velocity components. The total energy

$$
E=\rho\left(T+\frac{1}{2}\left(u^{2}+v^{2}+w^{2}\right)\right)
$$

and the pressure are related through the ideal gas law

$$
p=(\gamma-1) \rho T,
$$

where $T$ is the temperature field and $\gamma=c_{p} / c_{v}$ is the ratio between the heat capacities at constant pressure $\left(c_{p}\right)$ and volume $\left(c_{v}\right)$, respectively, and is assumed constant.

The viscous flux vectors are defined as

$$
\begin{aligned}
& \overline{\mathbf{F}}_{\nu}=J\left(\mathbf{F}_{\nu} \xi_{x}+\mathbf{G}_{\nu} \xi_{y}+\mathbf{H}_{\nu} \xi_{z}\right), \\
& \overline{\mathbf{G}}_{\nu}=J\left(\mathbf{F}_{\nu} \eta_{x}+\mathbf{G}_{\nu} \eta_{y}+\mathbf{H}_{\nu} \eta_{z}\right), \\
& \overline{\mathbf{H}}_{\nu}=J\left(\mathbf{F}_{\nu} \zeta_{x}+\mathbf{G}_{\nu} \zeta_{y}+\mathbf{H}_{\nu} \zeta_{z}\right),
\end{aligned}
$$


with

$$
\begin{gathered}
\mathbf{F}_{\nu}=\left[\begin{array}{c}
0 \\
\tau_{x x} \\
\tau_{y x} \\
\tau_{z x} \\
\tau_{x x} u+\tau_{y x} v+\tau_{z x} w+\frac{\gamma k}{\operatorname{Pr}} \frac{\partial T}{\partial x}
\end{array}\right], \mathbf{G}_{\nu}=\left[\begin{array}{c}
0 \\
\tau_{x y} \\
\tau_{y y} \\
\tau_{z y} \\
\tau_{x y} u+\tau_{y y} v+\tau_{z y} w+\frac{\gamma k}{\operatorname{Pr}} \frac{\partial T}{\partial y}
\end{array}\right] \\
\mathbf{H}_{\nu}=\left[\begin{array}{c}
0 \\
\tau_{x z} \\
\tau_{y z} \\
\tau_{z z} \\
\tau_{x z} u+\tau_{y z} v+\tau_{z z} w+\frac{\gamma k}{\operatorname{Pr}} \frac{\partial T}{\partial z}
\end{array}\right]
\end{gathered}
$$

Considering only Newtonian fluids, the stress tensor elements are given as

$$
\tau_{x_{i} x_{j}}=\mu\left(\frac{\partial u_{i}}{\partial x_{j}}+\frac{\partial u_{j}}{\partial x_{i}}\right)+\delta_{i j} \lambda \sum_{k=1}^{3} \frac{\partial u_{k}}{\partial x_{k}}
$$

where $\delta_{i j}$ is the Kronecker delta function. Here $\mu$ is the dynamic viscosity, $\lambda$ is the bulk viscosity, and $k$ is the coefficient of thermal conductivity. The velocity flux is obtained as

$$
\frac{\partial u_{i}}{\partial x_{j}}=\frac{\partial u_{i}}{\partial \xi} \xi_{x_{j}}+\frac{\partial u_{i}}{\partial \eta} \eta_{x_{j}}+\frac{\partial u_{i}}{\partial \zeta} \zeta_{x_{j}}
$$

and spatial derivatives of the temperature become

$$
\frac{\partial T}{\partial x_{i}}=\frac{\partial T}{\partial \xi} \xi_{x_{i}}+\frac{\partial T}{\partial \eta} \eta_{x_{i}}+\frac{\partial T}{\partial \zeta} \zeta_{x_{i}}
$$

The equations are normalized using the reference values $u_{\text {ref }}=u_{0}, \rho_{\text {ref }}=\rho_{0}, p_{\text {ref }}=$ $\rho_{0} u_{0}^{2}, T_{\text {ref }}=u_{0}^{2} / c_{v}$, and a reference length $L$, where $\left(\rho_{0}, u_{0}\right)$ is a given characteristic state. This gives a Reynolds number as $\operatorname{Re}=\rho_{0} u_{0} L / \mu_{0}$ and a Prandtl number as $\operatorname{Pr}=c_{p} \mu_{0} / k_{0}$. Note that the Reynolds number in (11), $\mathrm{Re}_{\mathrm{ref}}$, based on the reference values, in general is different from Re. In the remaining part of the paper we shall refer to the latter as the Reynolds number unless clarification is deemed necessary. With this normalization we need to specify the Mach number $M$, the Reynolds number Re, the length scale $L$, and a dimensional temperature $T_{0}$.

We consider only atmospheric air and take $\gamma=1.4$ and $\operatorname{Pr}=0.72$ in all problems to be considered later. To model the temperature dependence of the dynamic viscosity we use Sutherland's viscosity law [26]

$$
\frac{\mu(T)}{\mu_{s}}=\left(\frac{T}{T_{s}}\right)^{3 / 2} \frac{T_{s}+S}{T+S},
$$

where $\mu_{s}=1.716 \times 10^{-5} \mathrm{~kg} / \mathrm{m} \mathrm{sec}, T_{s}=273^{\circ} \mathrm{K}$, and $S=111^{\circ} \mathrm{K}$ for atmospheric air. Assuming that the Prandtl number is constant allows for modeling the temperature dependency of the coefficient of thermal conductivity similarly and we adopt Stokes's hypothesis (see, e.g., [26]) to obtain $\lambda=-\frac{2}{3} \mu$. 
4.1. The multidomain scheme. The aim is to develop a multidomain scheme for patching along the $\xi$-direction. We follow the approach developed in [1] and split the viscous fluxes into a parabolic term and two mixed terms

$$
\begin{aligned}
& \overline{\mathbf{F}}_{\nu}=\overline{\mathbf{F}}_{\xi}+\overline{\mathbf{F}}_{\eta}+\overline{\mathbf{F}}_{\zeta}=\bar{\Pi}_{\xi, \xi}+\bar{\Pi}_{\xi, \eta}+\bar{\Pi}_{\xi, \zeta}, \\
& \overline{\mathbf{G}}_{\nu}=\overline{\mathbf{G}}_{\eta}+\overline{\mathbf{G}}_{\xi}+\overline{\mathbf{G}}_{\zeta}=\bar{\Pi}_{\eta, \eta}+\bar{\Pi}_{\eta, \xi}+\bar{\Pi}_{\eta, \zeta}, \\
& \overline{\mathbf{H}}_{\nu}=\overline{\mathbf{H}}_{\zeta}+\overline{\mathbf{H}}_{\xi}+\overline{\mathbf{H}}_{\eta}=\bar{\Pi}_{\zeta, \zeta}+\bar{\Pi}_{\zeta, \xi}+\bar{\Pi}_{\zeta, \eta},
\end{aligned}
$$

where the general vector $\bar{\Pi}_{\alpha, \beta}=J \Pi_{\alpha, \beta}$ is defined as

$$
\Pi_{\alpha, \beta}=\frac{1}{\rho}\left[\begin{array}{c}
0 \\
\mu \nabla \alpha \cdot \nabla \beta \frac{\partial u}{\partial \beta}+\lambda \alpha_{x} \nabla \beta \cdot \mathbf{u}_{\beta}+\mu \beta_{x} \nabla \alpha \cdot \mathbf{u}_{\beta} \\
\mu \nabla \alpha \cdot \nabla \beta \frac{\partial v}{\partial \beta}+\lambda \alpha_{y} \nabla \beta \cdot \mathbf{u}_{\beta}+\mu \beta_{y} \nabla \alpha \cdot \mathbf{u}_{\beta} \\
\mu \nabla \alpha \cdot \nabla \beta \frac{\partial w}{\partial \beta}+\lambda \alpha_{z} \nabla \beta \cdot \mathbf{u}_{\beta}+\mu \beta_{z} \nabla \alpha \cdot \mathbf{u}_{\beta} \\
\mu(\nabla \beta \cdot \mathbf{u})\left(\nabla \alpha \cdot \mathbf{u}_{\beta}\right)+\lambda(\nabla \alpha \cdot \mathbf{u})\left(\nabla \beta \cdot \mathbf{u}_{\beta}\right)+(\nabla \alpha \cdot \nabla \beta)\left(\mu \mathbf{u} \cdot \mathbf{u}_{\beta}+\frac{\gamma k}{\operatorname{Pr}} \frac{\partial T}{\partial \beta}\right)
\end{array}\right] .
$$

Here we have introduced the vector

$$
\mathbf{u}_{\beta}=\left(\frac{\partial u}{\partial \beta}, \frac{\partial v}{\partial \beta}, \frac{\partial w}{\partial \beta}\right) .
$$

We continue by introducing the transformation derivative of the inviscid flux vector $\overline{\mathbf{F}}$ as

$$
\mathcal{A}=\frac{\partial \overline{\mathbf{F}}}{\partial \overline{\mathbf{q}}} .
$$

Linearizing $\mathcal{A}=\mathcal{A}\left(\mathbf{q}_{0}\right)$ allows for diagonalization through a similarity transform as $\Lambda=\mathcal{S}^{-1} \mathcal{A} \mathcal{S}$, where the right eigenvector matrix $\mathcal{S}$ and the left eigenvector matrix $\mathcal{S}^{-1}$ are given in the appendix.

The entries in the diagonal matrix $\Lambda$ are found as

$$
\lambda_{1}=\mathbf{u}_{0} \cdot \hat{\mathbf{n}}+c_{0}, \quad \lambda_{2}=\lambda_{3}=\lambda_{4}=\mathbf{u}_{0} \cdot \hat{\mathbf{n}}, \quad \lambda_{5}=\mathbf{u}_{0} \cdot \hat{\mathbf{n}}-c_{0},
$$

and correspond to the velocities of the characteristic waves for the Euler equations. Additionally, we obtain the characteristic functions $\overline{\mathbf{R}}=\mathcal{S}^{-1} \overline{\mathbf{q}}=J\left[R_{1}, R_{2}, R_{3}, R_{4}, R_{4}\right]^{T}$ as

$$
\overline{\mathbf{R}}=J\left[\begin{array}{l}
\left(\mathbf{m}-\rho \mathbf{u}_{0}\right) \cdot \hat{\mathbf{n}}+\frac{\gamma-1}{c_{0}}\left(E+\frac{1}{2} \rho \mathbf{u}_{0} \cdot \mathbf{u}_{0}-\mathbf{u}_{0} \cdot \mathbf{m}\right) \\
\left(m_{v}-\rho v_{0}\right) n_{1}-\left(m_{u}-\rho u_{0}\right) n_{2} \\
\rho-\frac{\gamma-1}{c_{0}^{2}}\left(E+\frac{1}{2} \rho \mathbf{u}_{0} \cdot \mathbf{u}_{0}-\mathbf{u}_{0} \cdot \mathbf{m}\right) \\
\left(m_{w}-\rho w_{0}\right) n_{1}-\left(m_{u}-\rho u_{0}\right) n_{3} \\
-\left(\mathbf{m}-\rho \mathbf{u}_{0}\right) \cdot \hat{\mathbf{n}}+\frac{\gamma-1}{c_{0}}\left(E+\frac{1}{2} \rho \mathbf{u}_{0} \cdot \mathbf{u}_{0}-\mathbf{u}_{0} \cdot \mathbf{m}\right)
\end{array}\right]
$$

where

$$
\mathbf{u}=(u, v, w), \quad \mathbf{m}=\left(m_{u}, m_{v}, m_{w}\right)=(\rho u, \rho v, \rho w), \quad c=\sqrt{\frac{\gamma p}{\rho}}
$$

is the velocity and momentum vector and the speed of sound, respectively. We have also introduced the unit vector $\hat{\mathbf{n}}$ pointing along $\nabla \xi$ as

$$
\hat{\mathbf{n}}=\left(n_{1}, n_{2}, n_{3}\right)=\frac{\nabla \xi}{\sqrt{\nabla \xi \cdot \nabla \xi}}=\frac{\left(\xi_{x}, \xi_{y}, \xi_{z}\right)}{\sqrt{\xi_{x}^{2}+\xi_{y}^{2}+\xi_{z}^{2}}} .
$$


The viscous patching vector $\overline{\mathbf{G}}$ introduced in [1] as a correction to the purely inviscid characteristic wave may likewise be derived in general curvilinear coordinates. Introducing the transformation derivative

$$
\mathcal{B}_{\alpha, \beta}=\mathcal{S}^{-1} \frac{\partial \bar{\Pi}_{\alpha, \beta}}{\partial \overline{\mathbf{q}}_{\beta}} \mathcal{S}
$$

with

$$
\overline{\mathbf{q}}_{\beta}=\frac{\partial \overline{\mathbf{q}}}{\partial \beta},
$$

we obtain, by straightforwardly generalizing the results obtained in [1], the viscous patching vector as

$$
\overline{\mathbf{G}}=\mathcal{B}_{\xi, \xi} \frac{\partial \overline{\mathbf{R}}}{\partial \xi}+\left(\mathcal{B}_{\xi, \eta}+\mathcal{B}_{\eta, \xi}\right) \frac{\partial \overline{\mathbf{R}}}{\partial \eta}+\left(\mathcal{B}_{\xi, \zeta}+\mathcal{B}_{\zeta, \xi}\right) \frac{\partial \overline{\mathbf{R}}}{\partial \zeta} .
$$

The explicit entries of $\mathcal{B}_{\alpha, \beta}$ may be found in the appendix. The entries of $\overline{\mathbf{G}}$ encompass information about the heat flux and the normal and tangential stress at the boundary.

We will note but not show that with this formulation it is possible to show that the full three-dimensional compressible Navier-Stokes equations on conservation form is symmetrizable even when given in general curvilinear coordinates. As this was the single most important property utilized in [1] we argue that the result establishing wellposedness and stability in Cartesian coordinates generalizes to curvilinear coordinates.

With the scheme successfully developed for Burgers's equation in mind, we are now ready to state the general scheme for solving the compressible Navier-Stokes equation using a multidomain approach. In each subdomain $\Omega^{k}$ we propose to solve

$$
\begin{aligned}
\frac{\partial \overline{\mathbf{q}}^{k}}{\partial t} & +\frac{\partial \overline{\mathbf{F}}^{k}}{\partial \xi^{k}}+\frac{\partial \overline{\mathbf{G}}^{k}}{\partial \eta^{k}}+\frac{\partial \overline{\mathbf{H}}^{k}}{\partial \zeta^{k}}=\frac{1}{\operatorname{Re}_{\mathrm{ref}}}\left(\frac{\partial \overline{\mathbf{F}}_{\nu}^{k}}{\partial \xi^{k}}+\frac{\partial \overline{\mathbf{G}}_{\nu}^{k}}{\partial \eta^{k}}+\frac{\partial \overline{\mathbf{H}}_{\nu}^{k}}{\partial \zeta^{k}}\right) \\
& -\tau_{1}^{k} Q_{k}^{-}\left(\xi_{i}^{k}\right) \mathcal{S}\left[\mathcal{R}_{k-1}^{-}\left(\overline{\mathbf{R}}_{0}^{k}-\overline{\mathbf{R}}_{N}^{k-1}\right)-\frac{1}{\operatorname{Re}_{\mathrm{ref}}} \mathcal{G}^{-}\left(\overline{\mathbf{G}}_{0}^{k}-\overline{\mathbf{G}}_{N}^{k-1}\right)\right] \\
& -\tau_{2}^{k} Q_{k}^{+}\left(\xi_{i}^{k}\right) \mathcal{S}\left[\mathcal{R}_{k}^{+}\left(\overline{\mathbf{R}}_{N}^{k}-\overline{\mathbf{R}}_{0}^{k+1}\right)+\frac{1}{\operatorname{Re}_{\mathrm{ref}}} \mathcal{G}^{+}\left(\overline{\mathbf{G}}_{N}^{k}-\overline{\mathbf{G}}_{0}^{k+1}\right)\right]
\end{aligned}
$$

where $\overline{\mathbf{R}}_{i}^{k}=\overline{\mathbf{R}}\left(\xi_{i}^{k}, \eta^{k}, \zeta^{k}\right)$ and $\overline{\mathbf{G}}_{i}^{k}=\overline{\mathbf{G}}\left(\xi_{i}^{k}, \eta^{k}, \zeta^{k}\right)$. To construct the correct boundary operator, we define the matrix operators

$$
\mathcal{R}_{k}^{-}=\left[\begin{array}{ccccc}
\lambda_{1}^{k} & 0 & 0 & 0 & 0 \\
0 & \lambda_{2}^{k} & 0 & 0 & 0 \\
0 & 0 & \lambda_{3}^{k} & 0 & 0 \\
0 & 0 & 0 & \lambda_{4}^{k} & 0 \\
0 & 0 & 0 & 0 & \alpha \lambda_{5}^{k}
\end{array}\right], \quad \mathcal{G}^{-}=\left[\begin{array}{ccccc}
1 & 0 & 0 & 0 & 0 \\
0 & 1 & 0 & 0 & 0 \\
0 & 0 & 1 & 0 & 0 \\
0 & 0 & 0 & 1 & 0 \\
0 & 0 & 0 & 0 & 1
\end{array}\right],
$$

where $\lambda_{i}^{k}=\lambda_{i}\left(\Gamma^{k}\right)$. We use $\alpha=0$ for subsonic conditions and $\alpha=1$ for supersonic inflow conditions. Likewise we define

$$
\mathcal{R}_{k}^{+}=\left[\begin{array}{ccccc}
0 & 0 & 0 & 0 & 0 \\
0 & 0 & 0 & 0 & 0 \\
0 & 0 & 0 & 0 & 0 \\
0 & 0 & 0 & 0 & 0 \\
0 & 0 & 0 & 0 & \beta\left|\lambda_{5}^{k}\right|
\end{array}\right], \quad \mathcal{G}^{+}=\left[\begin{array}{ccccc}
0 & 0 & 0 & 0 & 0 \\
0 & 1 & 0 & 0 & 0 \\
0 & 0 & 1 & 0 & 0 \\
0 & 0 & 0 & 1 & 0 \\
0 & 0 & 0 & 0 & 1
\end{array}\right],
$$

where $\beta=1$ for subsonic conditions and $\beta=0$ for supersonic outflow conditions. 
Note that the scheme given in (14) assumes that the velocity $\left(\mathbf{u}_{0} \cdot \hat{\mathbf{n}}\right)$ is positive, i.e., inflow at $\Gamma^{k-1}$ and outflow at $\Gamma^{k}$. However, in [1] stability was proven at inflow and outflow independently, and we may thus choose any combination of inflowoutflow interface conditions consistent with the flow realization while maintaining the asymptotic stability.

Similar to what we noted for the scheme for Burgers's equations, we observe that continuity of the characteristic functions and the viscous correction vector is enforced only weakly.

In order to complete the description of the scheme we need to choose the penalty parameters $\tau_{1}^{k}$ and $\tau_{2}^{k}$ such that the scheme is asymptotically stable. By noting that the proposed scheme in each subdomain is equivalent to the one-domain scheme discussed in [1], we argue that asymptotic stability is ensured by choosing the penalty parameters according to Lemma 5.2 in [1]. Thus, we use

$$
\begin{array}{ll}
\text { subsonic and supersonic inflow : } & \tau_{1}^{k} \geq \frac{1}{\omega \kappa}(1+\kappa-\sqrt{1+\kappa}) \frac{2}{L_{k}}, \\
\text { subsonic outflow : } & \tau_{2}^{k} \geq \frac{1}{\omega \kappa}(1+\kappa-\sqrt{1+\kappa}) \frac{2}{L_{k}}, \\
\text { supersonic outflow : } & \tau_{2}^{k} \geq \frac{1}{\omega}\left(1-\sqrt{\frac{1}{\kappa}}\right) \frac{2}{L_{k}},
\end{array}
$$

where

$$
\omega=\left(\frac{1}{N^{k}}\right)^{2}, \quad \kappa=\frac{1}{2 \omega} \frac{\gamma k_{0}}{\operatorname{Re}_{\mathrm{ref}} \operatorname{Pr} \rho_{0}\left|\mathbf{u}_{0} \cdot \hat{\mathbf{n}}\right|},
$$

and $L_{k}$ is the length of the $k$ th subdomain along $\xi$.

Although the proof in [1] was done for Legendre methods, we showed by numerical experiments that this result carries over to Chebyshev methods. Alternatively, the proposed scheme can be implemented as a Chebyshev-Legendre method, as introduced by Don and Gottlieb [27], thereby maintaining the advantage of using the Chebyshev polynomials while at the same time establishing the asymptotic stability. Such an implementation may be proven stable using the techniques discussed in [27].

In the calculation of the characteristic functions (12) and the viscous correction vector (13) needed for the scheme given by (14), we use the value of the state vector at the previous time-step as the linearization variable at the subdomain boundaries.

Similar to what was found for Burgers's equation, we note that the scheme is local in space and that only a small number of operations, relative to what is needed for calculation of the complete flux, is required to perform the patching.

It is instructive to observe that the boundary conditions are enforced through the characteristic variables as proposed by Gottlieb, Gunzburger, and Turkel [25] for reasons of stability. One should also note that in the limit of vanishing viscosity, the interface conditions become equivalent to those proposed by Kopriva [5] for the Euler equations, although we enforce them in a different way.

At open boundaries we have to supply proper boundary conditions. In the notation of (14) these should have the form

$$
\overline{\mathbf{R}}_{N}^{-1}=\mathcal{S}^{-1} \overline{\mathbf{g}}_{1}, \overline{\mathbf{R}}_{0}^{K+1}=\mathcal{S}^{-1} \overline{\mathbf{g}}_{2},
$$

yielding conditions on the value of the state vector at the boundaries and

$$
\begin{aligned}
& \overline{\mathbf{G}}_{N}^{-1}=\mathcal{B}_{\xi, \xi} \mathcal{S}^{-1} \frac{\partial \overline{\mathbf{g}}_{1}}{\partial \xi}+\left(\mathcal{B}_{\xi, \eta}+\mathcal{B}_{\eta, \xi}\right) \mathcal{S}^{-1} \frac{\partial \overline{\mathbf{g}}_{1}}{\partial \eta}+\left(\mathcal{B}_{\xi, \zeta}+\mathcal{B}_{\zeta, \xi}\right) \mathcal{S}^{-1} \frac{\partial \overline{\mathbf{g}}_{1}}{\partial \zeta} \\
& \overline{\mathbf{G}}_{0}^{K+1}=\mathcal{B}_{\xi, \xi} \mathcal{S}^{-1} \frac{\partial \overline{\mathbf{g}}_{2}}{\partial \xi}+\left(\mathcal{B}_{\xi, \eta}+\mathcal{B}_{\eta, \xi}\right) \mathcal{S}^{-1} \frac{\partial \overline{\mathbf{g}}_{2}}{\partial \eta}+\left(\mathcal{B}_{\xi, \zeta}+\mathcal{B}_{\zeta, \xi}\right) \mathcal{S}^{-1} \frac{\partial \overline{\mathbf{g}}_{2}}{\partial \zeta}
\end{aligned}
$$


where $\overline{\mathbf{g}}_{1}=J \mathbf{g}_{1}(\xi, \eta, \zeta, t)$ and $\overline{\mathbf{g}}_{2}=J \mathbf{g}_{2}(\xi, \eta, \zeta, t)$ are used to introduce information about the gradients of the state vector outside of the boundary. In most cases, very little is known about this, and for exterior flows one is often forced to approximate the gradients by zero. In this case the boundary conditions become equivalent to those proposed in [1].

We have used an adaptive fourth-order Runge-Kutta scheme for integration of the Navier-Stokes equations. The global time-step $\Delta t$ is found as [29]

$$
\Delta t=\mathrm{CFL} \times \min _{i, j, l, k}\left[\left|\mathbf{v}^{k} \cdot \mathbf{u}^{k}\right|+c^{k} \sqrt{\mathbf{v}^{k} \cdot \mathbf{v}^{k}}+\frac{2 \gamma}{\operatorname{PrRe}_{\mathrm{ref}}} \frac{\mu^{k}}{\rho^{k}} \mathbf{v}^{k} \cdot \mathbf{v}^{k}\right]^{-1},
$$

where CFL represents a generalized CFL number and $\mathbf{u}^{k}=\mathbf{u}^{k}\left(\xi_{i}^{k}, \eta_{j}^{k}, \zeta_{l}^{k}\right), c^{k}=$ $c^{k}\left(\xi_{i}^{k}, \eta_{j}^{k}, \zeta_{l}^{k}\right), \mu^{k}=\mu^{k}\left(\xi_{i}^{k}, \eta_{j}^{k}, \zeta_{l}^{k}\right)$, and $\rho^{k}=\rho^{k}\left(\xi_{i}^{k}, \eta_{j}^{k}, \zeta_{l}^{k}\right)$ signify the local values of the velocity, the sound speed, the dynamic viscosity, and the density, respectively. We have defined the local curvilinear vector as

$$
\mathbf{v}^{k}=\frac{|\nabla \xi|}{\Delta_{i} \xi^{k}}+\frac{|\nabla \eta|}{\Delta_{j} \eta^{k}}+\frac{|\nabla \zeta|}{\Delta_{l} \xi^{k}}
$$

where $\Delta_{i} \xi^{k}, \Delta_{j} \eta^{k}$, and $\Delta_{l} \zeta^{k}$ are the grid size along the three coordinate axes with respect to the indices $(i, j, l)$. Also $|\nabla \xi|=\left(\left|\xi_{x}\right|,\left|\xi_{y}\right|,\left|\xi_{z}\right|\right)$ and likewise for $|\nabla \eta|$ and $|\nabla \zeta|$.

4.2. Example 1. Quasi-one-dimensional nozzle flows. As a first example of the performance of the patching scheme for the compressible Navier-Stokes equations, we consider the flow in a quasi-one-dimensional Laval nozzle. The dynamics of the fluid is then described by a simplified set of equations as

$$
\frac{\partial \mathbf{q}}{\partial t}+\frac{\partial \mathbf{F}}{\partial x}+\mathbf{H}=\frac{1}{\operatorname{Re}_{\mathrm{ref}}} \frac{\partial \mathbf{F}_{\nu}}{\partial x},|x| \leq 1, t>0
$$

Here we have

$$
\begin{gathered}
\mathbf{q}=\left[\begin{array}{c}
\rho A \\
\rho u A \\
E A
\end{array}\right], \mathbf{F}=\left[\begin{array}{c}
\rho u A \\
\left(\rho u^{2}+p\right) A \\
(E+p) u A
\end{array}\right], \\
0 \\
\mathbf{F}_{\nu}=\left[\begin{array}{c}
0 \\
A \tau_{x x} \\
A u \tau_{x x}+A \frac{\gamma k}{\operatorname{Pr}} \frac{\partial T}{\partial x}
\end{array}\right], \quad \mathbf{H}=\left[\begin{array}{c}
0 \\
-p \frac{d A}{d x} \\
0
\end{array}\right] .
\end{gathered}
$$

This set of equations is obtained from (11) by using $\xi=x$ and $\eta=\zeta=0$, thus cancelling all $v$ - and $w$-components and $\eta$ - and $\zeta$-derivatives. Additionally, we use that for a slowly varying area variation $A(x)$ the quasi-one-dimensional divergence of a vector function $\mathbf{f}=(f, 0)$ may be approximated by

$$
\nabla \cdot \mathbf{f}=\frac{\partial f A}{\partial x}
$$

As reference values for nondimensionalizing the equations, we use the values at the throat.

For this problem the wave speeds of the characteristic waves become

$$
\lambda_{1}=u_{0}+c_{0}, \quad \lambda_{2}=u_{0}, \quad \lambda_{3}=u_{0}-c_{0},
$$


and the characteristic functions (12) are given as

$$
\mathbf{R}=\mathcal{S}^{-1} \mathbf{q}=\left[\begin{array}{c}
R_{1} \\
R_{2} \\
R_{3}
\end{array}\right]=A(x)\left[\begin{array}{c}
\rho u-u_{0} \rho+\frac{\gamma-1}{c_{0}}\left(E+\frac{1}{2} \rho u_{0}^{2}-\rho u u_{0}\right) \\
\rho-\frac{\gamma-1}{c_{0}^{2}}\left(E+\frac{1}{2} \rho u_{0}^{2}-\rho u u_{0}\right) \\
-\left(\rho u-u_{0} \rho\right)+\frac{\gamma-1}{c_{0}}\left(E+\frac{1}{2} \rho u_{0}^{2}-\rho u u_{0}\right)
\end{array}\right] .
$$

For inviscid flows, the three characteristic functions correspond to a copropagating sound wave, an entropy wave, and a counter propagating sound wave, respectively. For simulations of inviscid flows, specification of these characteristic functions whenever they enter the computational domain leads to a well-posed problem.

The viscous correction vector $\mathbf{G}$ from (13) becomes

$$
\mathbf{G}=\left[\begin{array}{c}
G_{1} \\
G_{2} \\
G_{3}
\end{array}\right]=\frac{1}{2 \rho_{0}}\left[\begin{array}{c}
\frac{k_{0}(\gamma-1)}{\operatorname{Pr}} \frac{\partial \zeta_{1}}{\partial x}+\left(\lambda_{0}+2 \mu_{0}\right) \frac{\partial \zeta_{2}}{\partial x} \\
-\frac{k_{0}(\gamma-1)}{c_{0} \operatorname{Pr}} \frac{\partial \zeta_{1}}{\partial x} \\
\frac{k_{0}(\gamma-1)}{\operatorname{Pr}} \frac{\partial \zeta_{1}}{\partial x}-\left(\lambda_{0}+2 \mu_{0}\right) \frac{\partial \zeta_{2}}{\partial x}
\end{array}\right],
$$

where we introduce

$$
\zeta_{1}=R_{1}+R_{3}-\frac{2 c_{0}}{\gamma-1} R_{2}, \quad \zeta_{2}=R_{1}-R_{3} .
$$

The terms with $\partial \zeta_{1} / \partial x$ is a consequence of the normal heat flux at the boundary, while $\partial \zeta_{2} / \partial x$ accounts for the normal stress.

4.2.1. Numerical tests. As a test case for the proposed multidomain scheme we have chosen a symmetric converging-diverging Laval nozzle with a cross-sectional area variation given as

$$
A(x)=1-0.8 x(1-x) \quad, \quad 0 \leq x \leq 1,
$$

and a ratio between the stagnation pressure and the back pressure of 0.78 . This results in a choked flow through the nozzle with the supersonic flow being terminated by a stationary shock in the divergent part of the nozzle. In the inviscid limit, this problem has an analytic solution (see, e.g., [28]) containing a shock at $x \simeq 0.773$ with a shock Mach number, $M_{s}=1.32$.

We have chosen the length of the nozzle $L=0.1 \mathrm{~m}$, the stagnation temperature $T_{0}=300^{\circ} \mathrm{K}$, and $M=1.0$ as the flow is choked.

Although the transonic nozzle flow leads to a steady state solution, we have implemented the scheme as for a fully unsteady problem using a fourth-order RungeKutta method. Additionally, we have in all simulations applied an exponential filter with $\gamma=10$ and $N_{c}=0$ to the solution to allow for increasing the timestep.

As initial condition we use the inviscid solution, smoothed by a fourth-order exponential filter; i.e., it is far from the steady state solution. As boundary conditions at the open inflow and outflow boundaries we use the inviscid solution, which, at least at high Reynolds numbers, is a very good approximation.

In Fig. 4(a) we show the steady state solutions obtained for $R e=100$ and $R e=$ 250 , and we observe good agreement between the one- and the four-domain solutions. As a way of testing the accuracy and asymptotic stability of the multidomain scheme we calculate the residual in the weighted $L_{2}$-norm (1) as

$$
\operatorname{Residual}(f(t+\Delta t))=\frac{\|f(t+\Delta t)-f(t)\|^{2}}{\|f(t)\|^{2}} .
$$


(a)

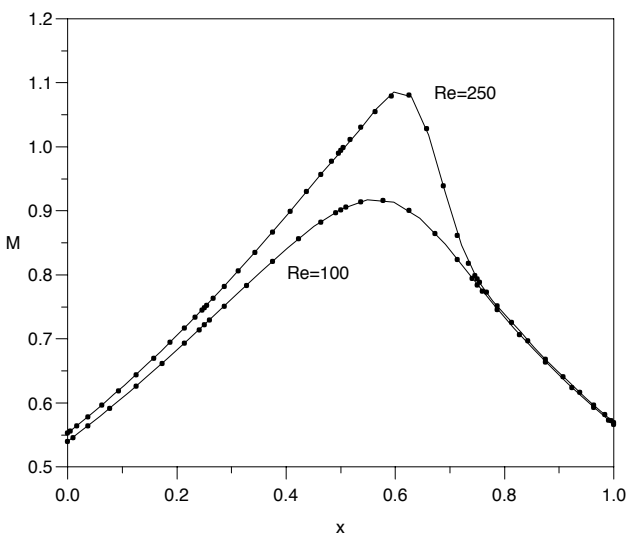

(b)

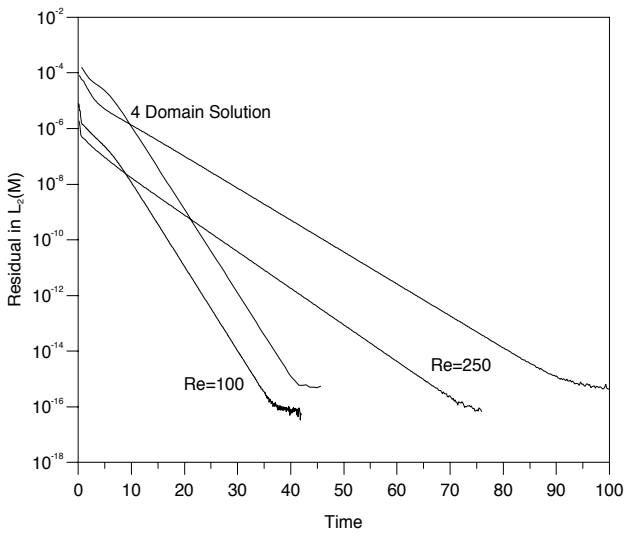

FIG. 4. (a) Steady state solution of the Mach profile for the viscous transonic nozzle flow. For the one-domain solutions (full line) at $\mathrm{Re}=100, N=32$ collocation points were used, whereas $N=48$ were used at $\mathrm{Re}=250$. The solutions symbolized by the dots represent the four-domain solution with the subdomains being equally sized and with $N / 4$ modes in each. CFL $=3.0$ was used in all simulations. (b) History of convergence of the Mach number for the one-and the four-domain solutions.

In Fig. 4(b) we show the corresponding residuals of the Mach number. The results for the other variables are similar. We find that the one domain as well as the multidomain solution converges to machine precision with the same rate of convergence. The slight difference in the actual physical time of convergence is a consequence of the accuracy of the initial approximation. As we use the same total number of modes in the onedomain and the four-domain solutions and both solutions converge at approximately the same physical time, we obtain a significant decrease in wallclock time by employing the multidomain approach. In this case the multidomain scheme is more than 10 times faster than the one-domain approach.

As final evidence of the performance of the scheme, we show in Fig. 5 the steady state solutions of the transonic nozzle flow at increasing Reynolds number, compared with the analytic purely inviscid solution. All viscous solutions are obtained using a five-domain solution, with the domains clustered around the viscous shock.

We observe that for low Reynolds number, the flow becomes purely subsonic and consequently the Mach profile changes upstream as well as downstream of the inviscid shock. For transonic flows, the steady state profiles are similar to the inviscid solution except in the highly viscous region in the neighborhood of the shock. For high Reynolds numbers $(\operatorname{Re} \geq 500)$ we find that the solution converges to the inviscid solution as $\mathrm{Re}^{-1 / 2}$, as expected. All viscous steady state profiles are computed with an $L_{2}$-residual less than $10^{-10}$.

4.3. Example 2. Flow around a circular cylinder. As a second test case for validating the proposed scheme, we have chosen unsteady compressible flow around an infinitely long circular cylinder. This flow is one of the most well-documented examples of simple exterior flows for which there exists an abundant amount of experimental results (see [30] and references therein). For $\operatorname{Re}<6$, where Re is based on the free-stream values of the state vector and the diameter of the cylinder, the flow is completely dominated by viscous effects and the flow pattern remains symmetric. 


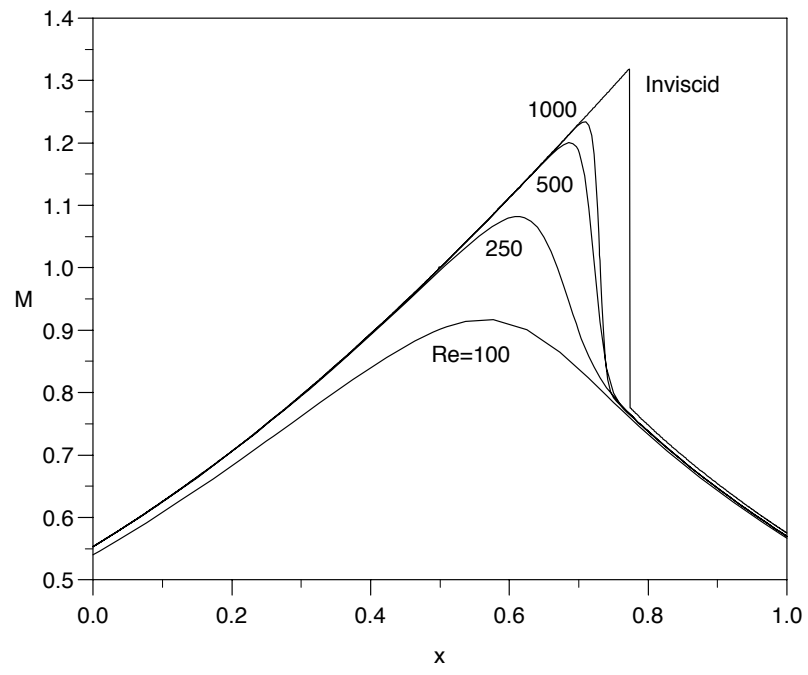

FIG. 5. Steady state Mach profile for the transonic viscous nozzle flow at $\operatorname{Re}=100,250,500$, 1000 and $\operatorname{Re}=\infty$. All solutions for finite Reynolds number are obtained as five-domain solutions with $C F L=3.0$.

Increasing Re leads to separation of the flow rear of the cylinder and a symmetric pair of separation bubbles appear. For Re $>44$ an instability is excited, resulting in a laminar flow pattern where vortices are shed alternately and periodically. For $\operatorname{Re}<180$ this pattern is very structured and the vortices retain their size and spacing downstream of the cylinder. The flow may, however, still be considered strictly twodimensional and is known as the von Karman street. Increasing Re further leads to the appearance of three-dimensional effects and finally, at very high Re, the flow becomes fully turbulent. Thus, despite the geometric simplicity of the flow, the observed flow patterns may be very complex.

We wish to simulate the unsteady flow in the von Karman shedding region and it is therefore sufficient to develop a two-dimensional model. The dynamics of the flow is described by the two-dimensional, compressible Navier-Stokes equations as given in (11), which we have normalized using the free-stream values of the flow. To simulate the dynamics of the flow we apply a multidomain approach, where the full computational domain $\Omega$ is constructed by several nonoverlapping concentric annular subdomains.

Each annular subdomain is mapped onto a rectangular computational domain $(\xi, \eta) \in[0,2 \pi] \times[-1,1]$. The branch cut, across which periodicity is enforced, is chosen at $\xi=0$, such that the physical grid relates to the computational grid as

$$
x=r(\eta) \cos \theta(\xi), \quad y=r(\eta) \sin \theta(\xi),
$$

where $(x, y)$ are the Cartesian coordinates, $(r, \theta)$ are the corresponding polar coordinates, and $(\xi, \eta)$ are the general curvilinear coordinates. Here $r(\eta)$ simply maps the standard interval $[-1,1]$ onto the radial extension of the grid whereas we have applied an azimuthal mapping [31] as

$$
\theta(\xi)=\tan ^{-1}\left[\frac{\left(1-\beta^{2}\right) \sin \xi}{\left(1+\beta^{2}\right) \cos \xi-2 \beta}\right] .
$$


This mapping has the effect of clustering the grid points around $\xi=0$, with the amount of clustering being controlled by $|\beta| \leq 1$. As a consequence of the geometry of the problem it is natural to choose a Fourier collocation method in $\xi$ and a Chebyshev collocation scheme in $\eta$.

By writing the problem in general curvilinear coordinates we obtain that although we treat a two-dimensional problem, we need only to give boundary conditions and perform domain patching in the $\eta$-direction as boundary conditions in $\xi$ are given through periodicity. Thus, the proposed one-dimensional approach for patching may be applied when solving this problem.

Following the approach developed in the previous sections, we need to derive the inviscid and viscous patching vectors for this particular case. From the general expressions given in section 4 we obtain the characteristic functions, (12)

$$
\overline{\mathbf{R}}=J\left[\begin{array}{l}
\left(\mathbf{m}-\rho \mathbf{u}_{0}\right) \cdot \hat{\mathbf{n}}+\frac{\gamma-1}{c_{0}}\left(E+\frac{1}{2} \rho \mathbf{u}_{0} \cdot \mathbf{u}_{0}-\mathbf{u}_{0} \cdot \mathbf{m}\right) \\
\left(m_{v}-\rho v_{0}\right) n_{1}-\left(m_{u}-\rho u_{0}\right) n_{2} \\
\rho-\frac{\gamma-1}{c_{0}^{2}}\left(E+\frac{1}{2} \rho \mathbf{u}_{0} \cdot \mathbf{u}_{0}-\mathbf{u}_{0} \cdot \mathbf{m}\right) \\
-\left(\mathbf{m}-\rho \mathbf{u}_{0}\right) \cdot \hat{\mathbf{n}}+\frac{\gamma-1}{c_{0}}\left(E+\frac{1}{2} \rho \mathbf{u}_{0} \cdot \mathbf{u}_{0}-\mathbf{u}_{0} \cdot \mathbf{m}\right)
\end{array}\right]
$$

where

$$
\hat{\mathbf{n}}=\left(n_{1}, n_{2}\right)=\frac{\nabla \eta}{\sqrt{\nabla \eta \cdot \nabla \eta}}=\frac{\left(\eta_{x}, \eta_{y}\right)}{\sqrt{\eta_{x}^{2}+\eta_{y}^{2}}}
$$

is a unit vector pointing in the radial direction. The four corresponding eigenvalues are

$$
\lambda_{1}=\mathbf{u}_{0} \cdot \hat{\mathbf{n}}+c_{0}, \quad \lambda_{2}=\lambda_{3}=\mathbf{u}_{0} \cdot \hat{\mathbf{n}}, \quad \lambda_{4}=\mathbf{u}_{0} \cdot \hat{\mathbf{n}}-c_{0},
$$

yielding the wave speed of the copropagating sound wave, the vorticity wave, the entropy wave, and the counter-propagating sound wave, respectively.

Following (13) we obtain the viscous patching vectors $\overline{\mathbf{G}}=J\left(G_{1}, G_{2}, G_{3}, G_{4}\right)^{T}$ as

$$
\begin{aligned}
G_{1} & =\frac{1}{\rho_{0} r}\left[\frac{(\gamma-1) k_{0}}{2 \operatorname{Pr}} \frac{\partial\left(r \zeta_{1}\right)}{\partial r}+\frac{2}{3} \mu_{0} \frac{\partial\left(r \zeta_{2}\right)}{\partial r}-\frac{1}{3} \mu_{0} \frac{\partial R_{2}}{\partial \theta}\right], \\
G_{2} & =\frac{\mu_{0}}{\rho_{0} r}\left[\frac{\partial\left(r R_{2}\right)}{\partial r}-\frac{1}{6} \frac{\partial \zeta_{2}}{\partial \theta}\right], \\
G_{3} & =-\frac{(\gamma-1) k_{0}}{\operatorname{Pr}} \frac{1}{2 c_{0} \rho_{0}} \frac{1}{r} \frac{\partial\left(r \zeta_{1}\right)}{\partial r}, \\
G_{4} & =\frac{1}{\rho_{0} r}\left[\frac{(\gamma-1) k_{0}}{2 \operatorname{Pr}} \frac{\partial\left(r \zeta_{1}\right)}{\partial r}-\frac{2}{3} \mu_{0} \frac{\partial\left(r \zeta_{2}\right)}{\partial r}+\frac{1}{3} \mu_{0} \frac{\partial R_{2}}{\partial \theta}\right],
\end{aligned}
$$

where, for convenience, we have introduced the symbols

$$
\zeta_{1}=R_{1}+R_{4}-\frac{2 c_{0}}{\gamma-1} R_{3}, \quad \zeta_{2}=R_{1}-R_{4}
$$

The terms associated with $\zeta_{1}$ account for effects of the normal heat flux across the boundary, while the remaining terms are related to the normal and tangential stress at the boundary. 


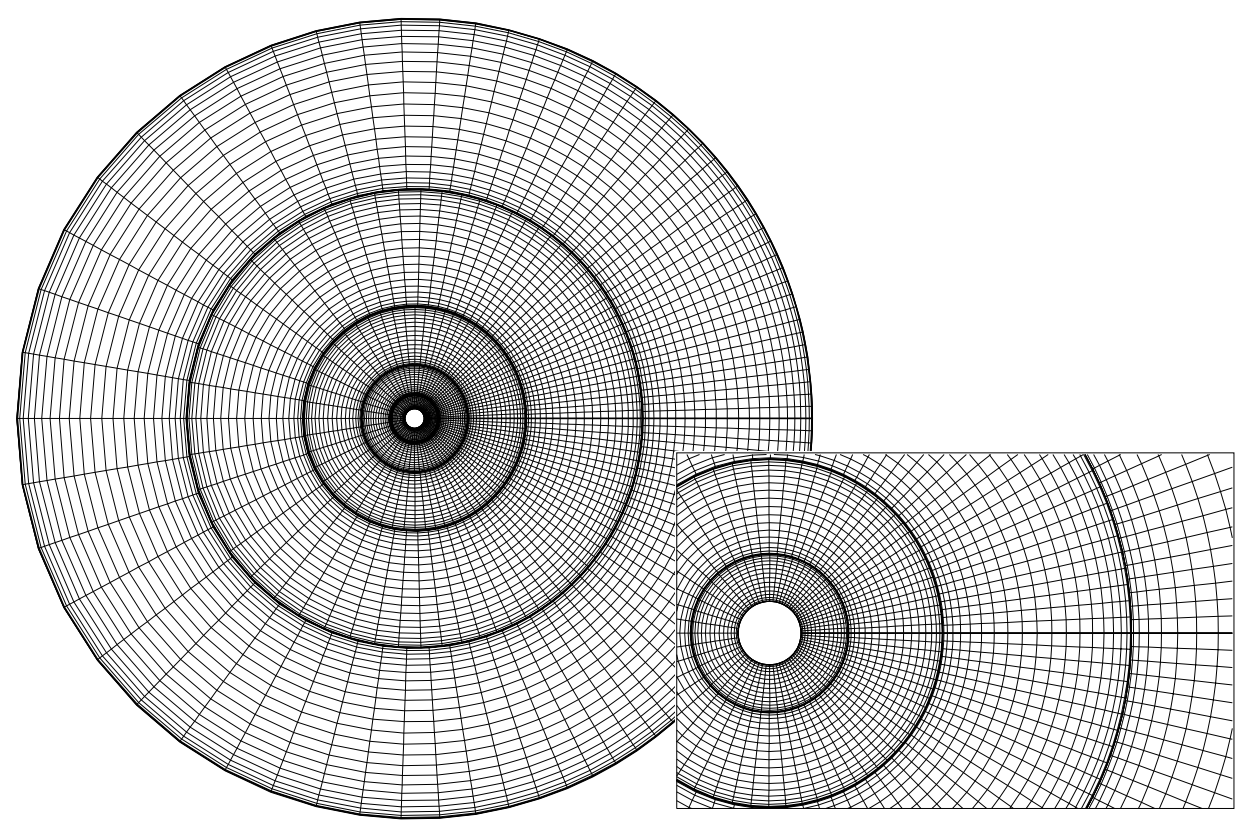

FIG. 6. Five-domain grid used for computation of flow around a cylinder in a computational domain of 20 cylinder diameters $(L)$. The subdomain boundaries are positioned at $r=1.25 L, r=$ $2.75 \mathrm{~L}, r=5.75 \mathrm{~L}$, and $r=11.75 \mathrm{~L}$. The box shows a blowup of the grid close to the cylinder.

The radial and azimuthal derivatives of a function $f(\xi, \eta)$ are found as

$$
\frac{\partial f}{\partial r}=\frac{\partial f}{\partial \eta} \frac{\partial \eta}{\partial r}, \quad \frac{\partial f}{\partial \theta}=\frac{\partial f}{\partial \xi} \frac{\partial \xi}{\partial \theta} .
$$

At the subdomain boundaries we use the values of the state vector at the previous time-step as linearization parameters and at the open boundary $\Gamma_{K}$ we use the freestream values. At the solid cylinder wall we assume no-slip, isothermal boundaries, i.e., $\mathbf{q}\left(\Gamma_{0}\right)=\left(\rho, 0,0, \rho T_{\infty}\right)^{T}$, where $\rho$ is determined numerically.

The solution has been integrated using an adaptive, explicit fourth-order RungeKutta with the boundary conditions and the subdomain patching being enforced at intermediate time-steps, where we also enforce continuity of the global solution and apply a filter with $N_{c}=0$ and $\gamma=10$. All simulations to be presented were done with $\mathrm{CFL}=3.0$.

4.3.1. Numerical tests. We have performed tests with a cylinder of diameter $L=0.1 \mathrm{~m}$, a free-stream Mach number $M=0.2$, and a stagnation temperature $T_{0}=300^{\circ} \mathrm{K}$.

In Fig. 6 we show a typical grid layout used for simulating the unsteady compressible flow around a cylinder. Note that the grids in the domains are nonconforming and we use periodic cubic spline interpolation between the different grids. The accuracy of this approach has been tested by comparing the results with Fourier interpolation, which is possible to use only in the absence of the mapping, and the spline interpolation is found to introduce only negligible errors. Using this grid, we have performed simulations at various Reynolds numbers. In Fig. 7 we show contour plots of the normalized density, normalized pressure, vorticity, and local Mach number. This clearly 

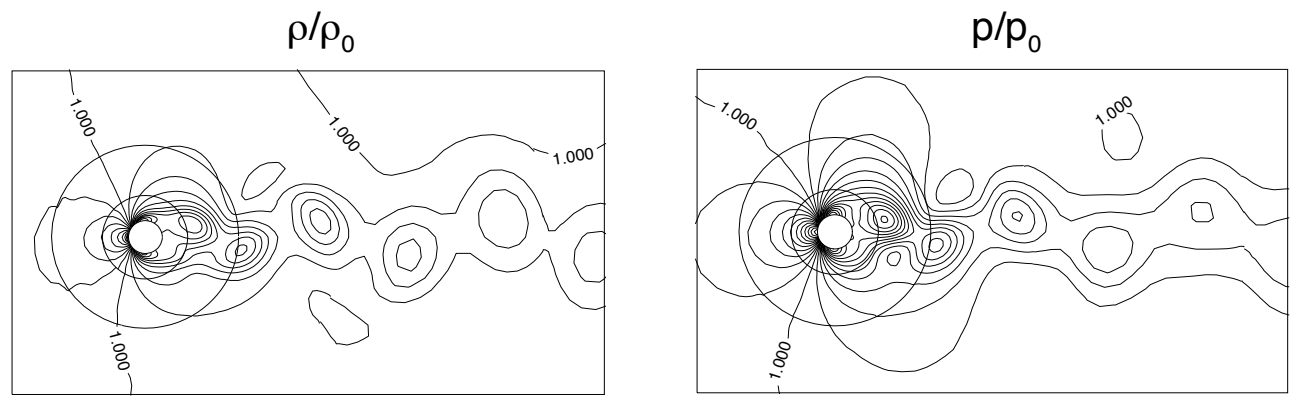

\section{Vorticity}

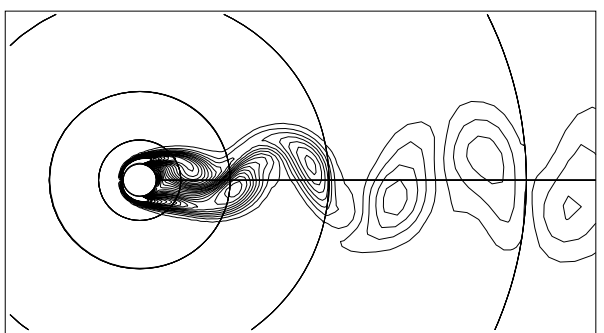

Mach

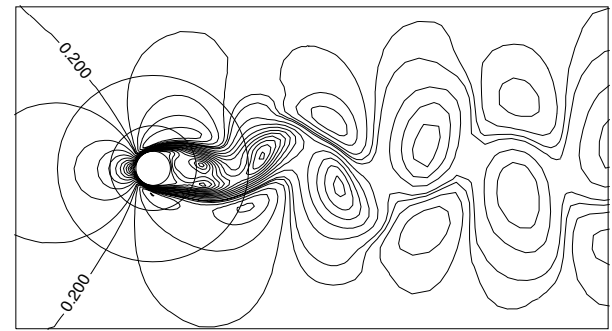

FIG. 7. Contour plot of the normalized density $\rho / \rho_{0}$, the normalized pressure $p / p_{0}$, the vorticity $\nabla \times \mathbf{u}$, and the local Mach number at $T=107$, corresponding to 18 shedding cycles at $\mathrm{Re}=100$. The circles represent the subdomain boundaries.

TABLE 2

Comparison of Strouhal number computed and reported from experiments [30].

\begin{tabular}{||c||c|c||}
\hline $\operatorname{Re}$ & $\begin{array}{c}S_{t} \\
\text { computed }\end{array}$ & $\begin{array}{c}S_{t} \\
\text { experiment [30] }\end{array}$ \\
75 & 0.149 & 0.149 \\
100 & 0.165 & 0.164 \\
125 & 0.177 & 0.175 \\
\hline
\end{tabular}

shows the well-known von Karman street behind the cylinder. We observe that the contour lines are continuous across subdomain boundaries, and we note that the vortices propagate undisturbed across the subdomain boundaries without any reflections from the artificial boundaries. Continuity of the contour lines in the plot of the vorticity distribution confirms that the scheme enforces continuity of the first-order derivatives also.

To evaluate the performance of the algorithm quantitatively, we have performed several computations at various Reynolds numbers. In Table 2 we compare the computed Strouhal number, i.e., the nondimensional shedding frequency $S_{t}=\omega L / u_{0}$, with that found in experiments [30]. The Strouhal number is calculated from time traces of the pressure in the wake of the cylinder. We observe very close agreement between computational and experimental results.

These comparisons lead us to conclude that the scheme performs well and is applicable also for fully unsteady, multidimensional flows. 
5. Concluding remarks. In this paper we have presented a further development of the well-posed and asymptotically stable open boundary conditions, proposed in [1], such that these can be used also as interface conditions for patching across subdomain boundaries. This led us to propose a unified approach for dealing with open boundaries as well as subdomain boundaries for performing multidomain solutions of dissipative, wave-dominated problems. Special attention has been given to the compressible Navier-Stokes equations given on conservation form with several examples of the specific details of the implementation.

The multidomain scheme that we propose may be proven asymptotically stable and, as we have shown, is well suited for performing simulations of steady as well as unsteady compressible flows.

Although we have only considered one- and two-dimensional examples, we are confident that the scheme may be applied for three-dimensional problems as well. However, the analysis of a patching scheme, based on general hexahedrals, for threedimensional, compressible flows is more involved than discussed here and will be the subject of the final paper in this trilogy [32].

Appendix. Similarity transform and viscous transformation derivative for the three-dimensional, compressible Navier-Stokes equations in general curvilinear coordinates. Introduce the transformation derivative of the inviscid flux vector $\overline{\mathbf{F}}$ as

$$
\mathcal{A}=\frac{\partial \overline{\mathbf{F}}}{\partial \overline{\mathbf{q}}} .
$$

Linearizing $\mathcal{A}=\mathcal{A}\left(\mathbf{q}_{0}\right)$ allows for diagonalizing through a similarity transform as $\Lambda=\mathcal{S}^{-1} \mathcal{A} \mathcal{S}$, where the right eigenvector matrix $\mathcal{S}$ and the left eigenvector matrix $\mathcal{S}^{-1}$ are given as

$$
\begin{gathered}
\mathcal{S}=\left[\begin{array}{ccccc}
\alpha & 0 & 1 & 0 & \alpha \\
\alpha\left(u+c n_{1}\right) & -n_{2} & u & -n_{3} & \alpha\left(u-c n_{1}\right) \\
\alpha\left(v+c n_{2}\right) & n_{1} & v & 0 & \alpha\left(v-c n_{2}\right) \\
\alpha\left(w+c n_{3}\right) & 0 & w & n_{1} & \alpha\left(w-c n_{3}\right) \\
\alpha(H+c \mathbf{u} \cdot \hat{\mathbf{n}}) & v n_{1}-u n_{2} & \frac{1}{2} c^{2} M^{2} & w n_{1}-u n_{3} & \alpha(H-c \mathbf{u} \cdot \hat{\mathbf{n}})
\end{array}\right], \\
\mathcal{S}^{-1}=\left[\begin{array}{ccccc}
\beta\left(\frac{1}{2} c^{2} M^{2}-c \frac{\mathbf{u} \cdot \hat{\mathbf{n}}}{\gamma-1}\right) & -\beta\left(u-\frac{c n_{1}}{\gamma-1}\right) & -\beta\left(v-\frac{c n_{2}}{\gamma-1}\right) & -\beta\left(w-\frac{c n_{3}}{\gamma-1}\right) & \beta \\
u n_{2}-v n_{1} & -n_{2} & n_{1} & 0 & 0 \\
1-\frac{1}{2}(\gamma-1) M^{2} & \frac{\gamma-1}{c^{2}} u & \frac{\gamma-1}{c^{2}} v & \frac{\gamma-1}{c^{2}} w & -\frac{\gamma-1}{c^{2}} \\
u n_{3}-w n_{1} & -n_{3} & 0 & n_{1} & 0 \\
\beta\left(\frac{1}{2} c^{2} M^{2}+c \frac{\mathbf{u} \cdot \hat{\mathbf{n}}}{\gamma-1}\right) & -\beta\left(u+\frac{c n_{1}}{\gamma-1}\right) & -\beta\left(v+\frac{c n_{2}}{\gamma-1}\right) & -\beta\left(w+\frac{c n_{3}}{\gamma-1}\right) & \beta
\end{array}\right] .
\end{gathered}
$$

Here

$$
\alpha=\frac{1}{2 c}, \quad \beta=\frac{\gamma-1}{c},
$$

and

$$
H=\frac{E+p}{\rho}
$$

is the enthalpy. 
For the general viscous transformation derivative

$$
\mathcal{B}_{\alpha, \beta}=\mathcal{S}^{-1} \frac{\partial \bar{\Pi}_{\alpha, \beta}}{\partial \overline{\mathbf{q}}_{\beta}} \mathcal{S}
$$

with

$$
\overline{\mathbf{q}}_{\beta}=\frac{\partial \overline{\mathbf{q}}}{\partial \beta},
$$

we obtain the entries $\mathcal{B}_{i j}=\rho\left(\mathcal{B}_{\alpha, \beta}\right)_{i j}$,

$$
\begin{aligned}
\mathcal{B}_{11}=\mathcal{B}_{55}= & \frac{1}{2}\left[(\mu+\lambda)(\nabla \alpha \cdot \hat{\mathbf{n}})(\nabla \beta \cdot \hat{\mathbf{n}})+(\nabla \alpha \cdot \nabla \beta)\left(\mu+\frac{(\gamma-1) k}{\operatorname{Pr}}\right)\right], \\
\mathcal{B}_{12}=-\mathcal{B}_{52}= & \lambda(\nabla \alpha \cdot \hat{\mathbf{n}})\left(n_{1} \beta_{y}-n_{2} \beta_{x}\right)+\mu(\nabla \beta \cdot \hat{\mathbf{n}})\left(n_{1} \alpha_{y}-n_{2} \alpha_{x}\right), \\
\mathcal{B}_{13}=\mathcal{B}_{53}= & -(\nabla \alpha \cdot \nabla \beta) c \frac{k}{\operatorname{Pr}}, \\
\mathcal{B}_{14}=-\mathcal{B}_{54}= & \lambda(\nabla \alpha \cdot \hat{\mathbf{n}})\left(n_{1} \beta_{z}-n_{3} \beta_{x}\right)+\mu(\nabla \beta \cdot \hat{\mathbf{n}})\left(n_{1} \alpha_{z}-n_{3} \alpha_{x}\right), \\
\mathcal{B}_{15}=\mathcal{B}_{51}= & \frac{1}{2}\left[-(\mu+\lambda)(\nabla \alpha \cdot \hat{\mathbf{n}})(\nabla \beta \cdot \hat{\mathbf{n}})+(\nabla \alpha \cdot \nabla \beta)\left(-\mu+\frac{(\gamma-1) k}{\operatorname{Pr}}\right)\right], \\
\mathcal{B}_{21}=-\mathcal{B}_{25}= & \frac{1}{2}\left[\mu(\nabla \alpha \cdot \hat{\mathbf{n}})\left(n_{1} \beta_{y}-n_{2} \beta_{x}\right)+\lambda(\nabla \beta \cdot \hat{\mathbf{n}})\left(n_{1} \alpha_{y}-n_{2} \alpha_{x}\right)\right], \\
\mathcal{B}_{22}= & \mu(\nabla \alpha \cdot \nabla \beta)\left(n_{1}^{2}+n_{2}^{2}\right)+(\lambda+\mu)\left(n_{2} \alpha_{x}-n_{1} \alpha_{y}\right)\left(n_{2} \beta_{x}-n_{1} \beta_{y}\right), \\
\mathcal{B}_{24}= & \mu(\nabla \alpha \cdot \nabla \beta)\left(n_{2} n_{3}\right)+\lambda\left(n_{2} \alpha_{x}-n_{1} \alpha_{y}\right)\left(n_{3} \beta_{x}-n_{1} \beta_{z}\right) \\
& +\mu\left(n_{3} \alpha_{x}-n_{1} \alpha_{z}\right)\left(n_{2} \beta_{x}-n_{1} \beta_{y}\right), \\
\mathcal{B}_{31}=\mathcal{B}_{35}= & -(\nabla \alpha \cdot \nabla \beta) \frac{\gamma-1}{2 c} \frac{k}{\operatorname{Pr}}, \\
\mathcal{B}_{33}= & (\nabla \alpha \cdot \nabla \beta) \frac{k}{\operatorname{Pr}}, \\
\mathcal{B}_{41}=-\mathcal{B}_{45}= & \frac{1}{2}\left[\mu(\nabla \alpha \cdot \hat{\mathbf{n}})\left(n_{1} \beta_{z}-n_{3} \beta_{x}\right)+\lambda(\nabla \beta \cdot \hat{\mathbf{n}})\left(n_{1} \alpha_{z}-n_{3} \alpha_{x}\right)\right], \\
\mathcal{B}_{42}= & \mu(\nabla \alpha \cdot \nabla \beta)\left(n_{2} n_{3}\right)+\lambda\left(n_{3} \alpha_{x}-n_{1} \alpha_{z}\right)\left(n_{2} \beta_{x}-n_{1} \beta_{y}\right) \\
& +\mu\left(n_{2} \alpha_{x}-n_{1} \alpha_{y}\right)\left(n_{3} \beta_{x}-n_{1} \beta_{z}\right), \\
\mathcal{B}_{44} & \mu(\nabla \alpha \cdot \nabla \beta)\left(n_{1}^{2}+n_{3}^{2}\right)+(\lambda+\mu)\left(n_{3} \alpha_{x}-n_{1} \alpha_{z}\right)\left(n_{3} \beta_{x}-n_{1} \beta_{z}\right), \\
\mathcal{B}_{23}=\mathcal{B}_{32}= & \mathcal{B} 34=\mathcal{B}_{43}=0,
\end{aligned}
$$

where $\alpha=(\xi, \eta, \zeta)$ and $\beta=(\xi, \eta, \zeta)$ and the unit vector $\hat{\mathbf{n}}$ is defined as

$$
\hat{\mathbf{n}}=\left(n_{1}, n_{2}, n_{3}\right)=\frac{\nabla \xi}{\sqrt{\nabla \xi \cdot \nabla \xi}}=\frac{\left(\xi_{x}, \xi_{y}, \xi_{z}\right)}{\sqrt{\xi_{x}^{2}+\xi_{y}^{2}+\xi_{z}^{2}}} .
$$

Note that in the special case of orthogonal curvilinear coordinates we may use

$$
\nabla \xi \cdot \nabla \eta=\nabla \eta \cdot \nabla \zeta=\nabla \zeta \cdot \nabla \xi=0,
$$

to simplify the mixed terms $(\alpha \neq \beta)$ considerably.

Acknowledgment. Part of this work was done while visiting Brown University, Division of Applied Mathematics, during the fall of 1994. The author expresses his 
gratitude to Professor D. Gottlieb for many inspiring conversations and the opportunity of staying in the stimulating atmosphere at Brown University. Another part of the work was done while in residence at University of New Mexico, Department of Mathematics and Statistics. The author thanks Professor E. A. Coutsias for the opportunity of a rewarding stay. Useful comments by Dr. D. A. Kopriva, Florida State University, are also appreciated.

\section{REFERENCES}

[1] J. S. Hesthaven and D. Gottlieb, A stable penalty method for the compressible NavierStokes equations: I. Open boundary conditions, SIAM J. Sci. Comput., 17 (1996), pp. 579612.

[2] A. T. PATERA, A spectral element methods for fluid dynamics: Laminar flow in a channel expansion, J. Comput. Phys., 54 (1984), pp. 468-488.

[3] M. G. Machraeg And C. L. Streett, Improvements in spectral collocation discretization through a multiple domain technique, Appl. Numer. Math., 2 (1986), pp. 95-108.

[4] C. Canuto, M. Y. Hussaini, A. Quarteroni and T. A. Zang, Spectral Methods in Fluid Dynamics, Springer Ser. Comput. Phys., Springer-Verlag, New York, 1988.

[5] D. Kopriva, Multidomain spectral solution of the Euler gas-dynamics equations, J. Comput. Phys., 96 (1991), pp. 428-450.

[6] D. Kopriva And J. H. Kolias, A conservative staggered-grid Chebyshev multidomain method for compressible flows, J. Comput. Phys., 125 (1996), pp. 244-261.

[7] A. Quarteroni, Domain decomposition methods for systems of conservation laws, SIAM J. Sci. Statist. Comput., 11 (1990), pp. 1029-1052.

[8] D. Sidilkover And G. E. Karniadakis, Non-oscillatory spectral element Chebyshev method for shock wave calculations, J. Comput. Phys., 107 (1993), pp. 10-22.

[9] J. G. Giannakouros, D. Sidilkover and G. E. Karniadakis, Spectral element-FCT method for the one- and two-dimensional compressible Euler equations, Comput. Methods Appl. Mech. Engrg., 116 (1994), pp. 113-121.

[10] D. Kopriva, A multi-domain spectral method for viscous compressible flows, AIAA J., 31 (1993), pp. 3376-3384.

[11] P. HANLEY, A strategy for the efficient simulation of viscous compressible flows using a multidomain pseudospectral method, J. Comput. Phys., 108 (1993), pp. 153-158.

[12] D. Kopriva, Multidomain spectral solution of compressible viscous flows, J. Comput. Phys., 115 (1994), pp. 184-199.

[13] D. Gottlieb, M. Y. Hussaini, And S. A. Orszag, Introduction: Theory and applications of spectral methods, in Spectral Methods for Partial Differential Equations, R. Voigt, D. Gottlieb, and M. Y. Hussaini, ed., SIAM, Philadelphia, PA, 1984, pp. 1-54.

[14] D. Funaro, Polynomial Approximation of Differential Equations, Springer-Verlag, New York, 1992.

[15] D. Gottlieb And E. TAdmor, The CFL condition for spectral approximations to hyperbolic initial-boundary value problems, Math. Comp., 56 (1991), pp. 565-588.

[16] C. E. Wasberg, On the Chebyshev Collocation Approximation and Domain Decomposition, Tech. report 7032, Norwegian Defense Research Establishment, Norway, 1992.

[17] W. S. Don And A. Solomonoff, Accuracy enhancement for higher derivatives using Chebyshev collocation and a mapping technique, SIAM J. Sci. Comput., 16 (1995), pp. 1253-1268.

[18] D. Gottlieb, Private communication, 1994.

[19] R. Courant And D. Hilbert, Methods of Mathematical Physics, Vols. I and II, John Wiley, New York, 1989.

[20] D. Gottlieb And S. A. Orszag, Numerical Analysis of Spectral Methods: Theory and Applications, SIAM CBMS 26, SIAM, Philadelphia, PA, 1977.

[21] H. O. Kreiss and J. Lorenz, Initial-Boundary Value Problems and the Navier-Stokes Equations, Ser. Pure Appl. Math., Academic Press, San Diego, 1989.

22] I. BABUS̆KA AND M. SURI, The $p$ - and $h-p$ version of the finite element method. An overview, Comput. Methods Appl. Mech. Engrg., 80 (1990), pp. 5-26.

[23] C. Basdevant, M. Deville, P. Haldenwang, J. M. Lacroix, J. Ouazzani, R. Peyret, P. Orlandi, AND A. T. PATERA, Spectral and finite difference solutions of the Burgers equation, Comput. Fluids, 14 (1986), pp. 23-41.

[24] C. Mavriplis, Adaptive mech strategies for the spectral element method, Comput. Methods Appl. Mech. Engrg., 116 (1994), pp. 77-86. 
[25] D. Gottlieb, M. Gunzburger, And E. Turkel, On numerical boundary treatment of hyperbolic systems for finite difference and finite element methods, SIAM J. Numer. Anal., 19 (1982), pp. 671-682.

[26] H. Schlichting, Boundary-Layer Theory, 7th ed., McGraw-Hill, New York, 1979.

[27] W. S. Don And D. GotTlieb, The Chebyshev-Legendre method: Implementing Legendre methods on Chebyshev grids, SIAM J. Numer. Anal., 31 (1994), pp. 1519-1534.

[28] Ames Research Staff, Equations, Tables and Charts for Compressible Flow, NACA report 1135, Ames Aeronautical Laboratory, California, 1953.

[29] R. W. MacCormack And H. Lomax, Numerical solution of compressible viscous flows, Ann. Rev. Fluid Mech., 11 (1979), pp. 289-316.

[30] C. H. K. Williamson, Oblique and parallel modes of vortex shedding in the wake of a circular cylinder at low Reynolds numbers, J. Fluid Mech., 206 (1989), pp. 579-627.

[31] J. Augenbaum, An Adaptive Pseudospectral Method for Discontinuous Problems, NASA/ICASE report 88-54, NASA Langley Research Center, Hampton, VA, 1988.

[32] J. S. Hesthaven, A stable penalty method for the compressible Navier-Stokes equations: III. Multidimensional domain decomposition schemes, SIAM J. Sci. Comput., to appear. 